\title{
Inverse $K$-Chevalley formulas for semi-infinite flag manifolds, I: minuscule weights in ADE type
}

\author{
Takafumi Kouno ${ }^{1}$, Satoshi Naito ${ }^{2}$, Daniel Orr ${ }^{3}$ and Daisuke Sagaki ${ }^{4}$ \\ ${ }^{1}$ Department of Mathematics, Tokyo Institute of Technology, 2-12-1 Oh-okayama, Meguro-ku, Tokyo 152-8551, Japan \\ E-mail: kouno.t.ab@m.titech.ac.jp. \\ ${ }^{2}$ Department of Mathematics, Tokyo Institute of Technology, 2-12-1 Oh-okayama, Meguro-ku, Tokyo 152-8551, Japan \\ E-mail: naito@math.titech.ac.jp. \\ ${ }^{3}$ Department of Mathematics (MC 0123), 460 McBryde Hall, Virginia Tech, 225 Stanger St., Blacksburg, VA 24061, USA \\ E-mail: dorr@vt.edu. \\ ${ }^{4}$ Institute of Mathematics, University of Tsukuba, 1-1-1 Tennodai, Tsukuba, Ibaraki 305-8571, Japan \\ E-mail: sagaki@math.tsukuba.ac.jp.
}

Received: 03 December 2020; Accepted: 25 May 2021

2020 Mathematics Subject Classification: Primary - 20C08, 17B37; Secondary - 14N15, 14M15, 33D52, 81R10

\begin{abstract}
We prove an explicit inverse Chevalley formula in the equivariant $K$-theory of semi-infinite flag manifolds of simply laced type. By an 'inverse Chevalley formula' we mean a formula for the product of an equivariant scalar with a Schubert class, expressed as a $\mathbb{Z}\left[q^{ \pm 1}\right]$-linear combination of Schubert classes twisted by equivariant line bundles. Our formula applies to arbitrary Schubert classes in semi-infinite flag manifolds of simply laced type and equivariant scalars $e^{\lambda}$, where $\lambda$ is an arbitrary minuscule weight. By a result of Stembridge, our formula completely determines the inverse Chevalley formula for arbitrary weights in simply laced type except for type $E_{8}$. The combinatorics of our formula is governed by the quantum Bruhat graph, and the proof is based on a limit from the double affine Hecke algebra. Thus our formula also provides an explicit determination of all nonsymmetric $q$-Toda operators for minuscule weights in ADE type.
\end{abstract}

\section{Introduction}

Let $\mathbf{Q}_{G}^{\text {rat }}$ be the semi-infinite flag manifold. This is a reduced ind-scheme whose set of $\mathbb{C}$-valued points is $G(\mathbb{C}((z))) /(H(\mathbb{C}) \cdot N(\mathbb{C}((z))))$ (see [11] for details), where $G$ is a simply connected simple algebraic group over $\mathbb{C}, B=H N \subset G$ is a Borel subgroup, $H$ is a maximal torus and $N$ is the unipotent radical of $B$. For each affine Weyl group element $x \in W_{\mathrm{af}}=W \ltimes Q^{\vee}$, with $W=\left\langle s_{i} \mid i \in I\right\rangle$ the (finite) Weyl group and $Q^{\vee}=\bigoplus_{i \in I} \mathbb{Z} \alpha_{i}^{\vee}$ the coroot lattice of $G$, one has a semi-infinite Schubert variety $\mathbf{Q}_{G}(x) \subset \mathbf{Q}_{G}^{\text {rat }}$, which is infinite-dimensional and is given as an orbit closure for the Iwahori subgroup $\mathbf{I} \subset G(\mathbb{C}[[z]])$. We distinguish the semi-infinite Schubert variety $\mathbf{Q}_{G}:=\mathbf{Q}_{G}(e) \subset \mathbf{Q}_{G}^{\text {rat }}$ associated to the identity element $e$ of the affine Weyl group, and also call it the semi-infinite flag manifold.

Our main object of study is the equivariant $K$-group $K_{H \times \mathbb{C}^{*}}\left(\mathbf{Q}_{G}\right)$-and that of $\mathbf{Q}_{G}^{\text {rat }}$, denoted by $K_{H \times \mathbb{C}^{*}}\left(\mathbf{Q}_{G}^{\text {rat }}\right)$-which is a variant of the $K$-group $K_{\tilde{\mathbf{I}}}^{\prime}\left(\mathbf{Q}_{G}\right)$ introduced recently in [13]. Our $K$-group is a module over the equivariant scalar ring $\mathbb{Z}\left[q^{ \pm 1}\right][P]$, where $P$ is the weight lattice of $G, \mathbb{Z}[P]=\mathbb{Z}\left[e^{\mu}\right.$ : $\mu \in P]$ is the character ring of $H$ and $q \in R\left(\mathbb{C}^{*}\right)$ is the character of loop rotation. Therefore, the $K$-group $K_{H \times \mathbb{C}^{*}}\left(\mathbf{Q}_{G}\right)$ is a $\mathbb{Z}\left[q^{ \pm 1}\right][P]$-submodule of an extension of scalars of the equivariant $K$-group $K_{\tilde{\mathbf{I}}}^{\prime}\left(\mathbf{Q}_{G}\right)$ of [13] with respect to the Iwahori subgroup I and loop rotation.

(C) The Author(s), 2021. Published by Cambridge University Press. This is an Open Access article, distributed under the terms of the Creative Commons Attribution licence (http://creativecommons.org/licenses/by/4.0/), which permits unrestricted re-use, distribution, and reproduction in any medium, provided the original work is properly cited. 
A fundamental result of [13] is the combinatorial Chevalley formula for dominant weights in the $K$-group $K_{\tilde{\mathbf{I}}}^{\prime}\left(\mathbf{Q}_{G}\right)$ (and hence in $K_{H \times \mathbb{C}^{*}}\left(\mathbf{Q}_{G}\right)$ ). This formula describes, in terms of semi-infinite Lakshmibai-Seshadri paths, the tensor product of the class of the line bundle $\left[\mathcal{O}_{\mathbf{Q}_{G}}(\lambda)\right]$ associated to a dominant weight $\lambda \in P^{+}$with the class of the structure sheaf $\left[\mathcal{O}_{\mathbf{Q}_{G}(x)}\right]$ of a semi-infinite Schubert variety $\mathbf{Q}_{G}(x) \subset \mathbf{Q}_{G}$ for $x=w t_{\xi} \in W_{\text {af }}^{\geq 0}:=W \times Q^{\vee,+} \subset W_{\text {af }}$, where $Q^{\vee,+}:=\sum_{i \in I} \mathbb{Z}_{\geq 0} \alpha_{i}^{\vee} \subset Q^{\vee}$. This was followed up in [24] by another combinatorial Chevalley formula in $K_{H \times \mathbb{C}^{*}}\left(\mathbf{Q}_{G}\right)$, giving the tensor product of a Schubert class with an antidominant line bundle. The two Chevalley formulas-dominant [13] and antidominant [24]-were unified in [19], giving the general Chevalley formula in $K_{H \times \mathbb{C}^{*}}\left(\mathbf{Q}_{G}\right)$ for arbitrary weights $\lambda \in P$.

The Chevalley formulas of $[13,24,19]$ thus provide the complete analogue for semi-infinite flag manifolds of their previously well-understood $K$-theory counterparts for the standard Kac-Moody flag varieties [26, 22, 7, 20, 21]. In all such formulas, the objective is to expand the tensor product of a Schubert class with an equivariant line bundle, as a linear combination of Schubert classes with equivariant scalar coefficients. In the case of $\mathbf{Q}_{G}$, by [19], this takes the form

$$
\left[\mathcal{O}_{\mathbf{Q}_{G}(x)}(\lambda)\right]=\sum_{\substack{y \in W_{\text {af }}^{\geq 0} \\ \mu \in P}} c_{x, y}^{\lambda, \mu} \cdot e^{\mu} \cdot\left[\mathcal{O}_{\mathbf{Q}_{G}(y)}\right],
$$

with $x, y \in W_{\text {af }}^{\geq 0}, \lambda, \mu \in P$ and $c_{x, y}^{\lambda, \mu} \in \mathbb{Z}\left[q^{ \pm 1}\right]$. We note that the sum on the right-hand side of equation (1.1), while generally infinite, satisfies a notion of convergence introduced in [13].

In this paper, we shall study the inverse ${ }^{1}$ expansion in $K_{H \times \mathbb{C}^{*}}\left(\mathbf{Q}_{G}\right)$ :

$$
e^{\lambda} \cdot\left[\mathcal{O}_{\mathbf{Q}_{G}(x)}\right]=\sum_{\substack{y \in W_{\text {af }}^{\geq 0} \\ \mu \in P}} d_{x, y}^{\lambda, \mu} \cdot\left[\mathcal{O}_{\mathbf{Q}_{G}(y)}(\mu)\right]
$$

for $x \in W_{\mathrm{af}}^{\geq 0}$ and $\lambda \in P$. In contrast to equation (1.1), the expansion in equation (1.2) is finite, as established in [25] for simply laced $G$-namely, $d_{x, y}^{\lambda, \mu} \in \mathbb{Z}\left[q^{ \pm 1}\right]$ for any $\lambda, \mu \in P$ and $x, y \in W_{\text {af }}^{\geq 0}$, and the right-hand side of equation (1.2) is always a finite sum. (These properties are expected to hold for arbitrary $G$.)

We call any formula for the right-hand side of equation (1.2) an inverse Chevalley formula for $\lambda \in P$ in $K_{H \times \mathbb{C}^{*}}\left(\mathbf{Q}_{G}\right)$. The analogous expansion for finite-dimensional flag manifolds $G / B$ was studied by Mathieu [23] in the context of filtrations of $B$-modules (compare [23, p. 239]). In fact, by [10, Proof of Proposition 1.15], one has a surjective map of $\mathbb{Z}[P]$-modules given by

$$
\begin{aligned}
K_{H \times \mathbb{C}^{*}}\left(\mathbf{Q}_{G}\right) & \stackrel{\text { trunc }}{\longmapsto} K_{H}(G / B) \\
q & \longmapsto 1 \\
{\left[\mathcal{O}_{\mathbf{Q}_{G}(y)}\right] } & \longmapsto \begin{cases}{\left[\mathcal{O}_{X(y)}\right]} & \text { if } y \in W, \\
0 & \text { otherwise }\end{cases}
\end{aligned}
$$

where $\left[\mathcal{O}_{X(y)}\right]$ for $y \in W$ denotes the structure sheaf of the Schubert variety

$$
X(y)=\overline{B y w_{\circ} B / B} \subset G / B
$$

and $w_{\circ}$ is the longest element of $W$. The map trunc also respects the tensor product by equivariant

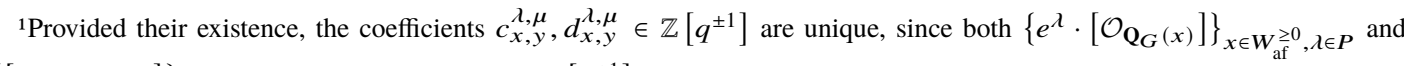
$\left\{\left[\mathcal{O}_{\mathbf{Q}_{G}(x)}(\lambda)\right]\right\}_{x \in W_{\text {af }} \geq 0, \lambda \in \boldsymbol{P}}$ are (topologically) $\mathbb{Z}\left[q^{ \pm 1}\right]$-linearly independent sets in $K_{\boldsymbol{H} \times \mathbb{C}^{*}}\left(\mathbf{Q}_{G}\right)$. Existence follows from $[13,24]$ for equation (1.1) and [25] for equation (1.2). 
line bundles. Thus, for $x \in W$, the image of equation (1.2) under trunc produces the analogous inverse Chevalley expansion in $K_{H}(G / B)$ (see also Remark 3.13). In this sense, any inverse Chevalley formula (1.2) must incorporate the necessary 'corrections' in $K_{H \times \mathbb{C}^{*}}\left(\mathbf{Q}_{G}\right)$ to the classical inverse Chevalley formulas in $K_{H}(G / B)$.

We note that in the case of $K_{H}(G / B)$, there is a simple transformation to pass between ordinary and inverse Chevalley formulas (as explained in, e.g., [23, p. 239]). The lack of such a transformation for $K_{H \times \mathbb{C}^{*}}\left(\mathbf{Q}_{G}\right)$ justifies the independent study of inverse Chevalley formulas in the semi-infinite setting. Moreover, the finiteness of equation (1.2) offers an advantage over equation (1.1).

The purpose of this paper is to prove a completely explicit, combinatorial inverse Chevalley formula in the equivariant $K$-group $K_{H \times \mathbb{C}^{*}}\left(\mathbf{Q}_{G}\right)$ in the case of a simply laced group $G$ and a minuscule weight $\lambda \in P$. Before stating our results more precisely, let us discuss further motivations for this work.

\subsection{Nil-DAHA and q-Heisenberg actions on $K_{H \times \mathbb{C}^{*}}\left(\mathbf{Q}_{G}^{\mathrm{rat}}\right)$}

One approach to understanding equations (1.1) and (1.2) is that they relate two actions of the group algebra $\mathbb{Z}[P]$ on $K_{H \times \mathbb{C}^{*}}\left(\mathbf{Q}_{G}^{\text {rat }}\right)$, one given by equivariant scalar multiplication (the left-hand side of equation (1.2)) and the other by the tensor product with equivariant line bundles (the left-hand side of equation (1.1)). These actions of $\mathbb{Z}[P]$ extend to that of two distinct algebras on $K_{H \times \mathbb{C}^{*}}\left(\mathbf{Q}_{G}^{\text {rat }}\right)$ : the nil double affine Hecke algebra (nil-DAHA) and a $q$-Heisenberg algebra.

Multiplication by equivariant scalars extends to a left action of the nil-DAHA $\mathbb{H}_{0}$ on $K_{H \times \mathbb{C}^{*}}\left(\mathbf{Q}_{G}^{\text {rat }}\right)$, in a way that is conceptually similar to the action of nil-Hecke algebras on the equivariant $K$-theory of Kac-Moody flag varieties [8]. On the right, however, instead of a nil-Hecke algebra, one has an action of a $q$-Heisenberg algebra $\mathfrak{H}$. This is generated by tensor products with equivariant line bundles $[\mathcal{O}(\lambda)]$ $(\lambda \in P)$ and translations $Q^{\vee} \cong H(\mathbb{C}((z))) / H(\mathbb{C}[[z]])$. The two actions commute, making $K_{H \times \mathbb{C}^{*}}\left(\mathbf{Q}_{G}^{\text {rat }}\right)$ an $\left(\mathbb{H}_{0}, \mathfrak{S}\right)$-bimodule.

This bimodule structure is a fundamental tool in the study of $K_{H \times \mathbb{C}^{*}}\left(\mathbf{Q}_{G}^{\text {rat }}\right)$, as standard methods such as localisation are not available. Furthermore, as explained in [25], it gives a geometric realisation of the nonsymmetric $q$-Toda system introduced in [4] (see [1, 2, 5] for related results on the usual $q$-Toda system and [14, 15] for its (q, t)-extension given by Macdonald difference operators in type $A$ ). For us, the fact that the $\mathbb{H}_{0}$-action on $K_{H \times \mathbb{C}^{*}}\left(\mathbf{Q}_{G}^{\text {rat }}\right)$ includes the operators of multiplication by equivariant scalars is key. We use the limit construction [25] of the $\mathbb{H}_{0}$-action on $K_{H \times \mathbb{C}^{*}}\left(\mathbf{Q}_{G}^{\text {rat }}\right)$ to find and prove our inverse Chevalley formula in $K_{H \times \mathbb{C}^{*}}\left(\mathbf{Q}_{G}\right)$, which is given by Theorem 1 .

\subsection{Quantum K-theory of $G / B$}

In $[10,12]$, Kato established a $\mathbb{Z}[P]$-module isomorphism - with $\mathbb{Z}[P]$ acting by equivariant scalars from the (completed) $H$-equivariant quantum $K$-group $Q K_{H}(G / B):=K_{H}(G / B) \otimes \mathbb{Z}\left[\left[Q^{\vee,+}\right]\right]$ of the finite-dimensional flag manifold $G / B$ onto the $H$-equivariant $K$-group $K_{H}\left(\mathbf{Q}_{G}\right)$ obtained by specialising $q=1$ in $K_{H \times \mathbb{C}^{*}}\left(\mathbf{Q}_{G}\right)$. (Here $\mathbb{Z}\left[\left[Q^{\vee,+}\right]\right]$ is the ring of formal power series in the (Novikov) variables $Q_{i}, i \in I$.) Kato's isomorphism respects Schubert classes and intertwines the quantum multiplication in $Q K_{H}(G / B)$ with the tensor product by line bundles in $K_{H}\left(\mathbf{Q}_{G}\right)$. Thus it provides a means to transport formulas from $K_{H}\left(\mathbf{Q}_{G}\right)$ to $Q K_{H}(G / B)$. In the sequel [16] to this paper, we will use Kato's isomorphism to derive a corresponding inverse Chevalley formula in $Q K_{H}(G / B)$.

\subsection{Our results}

Let us now explain our results in more detail. Recall that a weight $\lambda \in P$ is called minuscule if $\left\langle\lambda, \alpha^{\vee}\right\rangle \in\{0, \pm 1\}$ for all $\alpha \in \Delta$. Nonzero minuscule weights exist in all types except $E_{8}, F_{4}$ and $G_{2}$.

Our results explicitly describe the inverse Chevalley formula in $K_{H \times \mathbb{C}^{*}}\left(\mathbf{Q}_{G}\right)$ for arbitrary minuscule weights $\lambda \in P$ in the case when $G$ is simply laced. By iteration, our formulas completely determine the inverse Chevalley rule for arbitrary weights in $\mathrm{ADE}$ type (except in type $E_{8}$ ). Indeed, a result of 
Stembridge [27] (as stated in [18, Theorem 2.1]) asserts that in all types except $E_{8}, F_{4}$ and $G_{2}$, the minuscule weights form a set of generators for the weight lattice. (The full version of Stembridge's result, which holds in arbitrary type, requires quasi-minuscule weights. We plan to take up the study of our constructions in the case of quasi-minuscule weights elsewhere.)

Any minuscule weight belongs to the Weyl group orbit of a dominant minuscule weight - that is, a minuscule fundamental weight. Suppose $\varpi_{k} \in P^{+}$is a minuscule fundamental weight. Set $J=I \backslash\{k\}$, and consider the parabolic subgroup $W_{J}=\left\langle s_{j} \mid j \in J\right\rangle$, which is the stabiliser of $\varpi_{k}$. Let $W^{J}$ be the set of minimal coset representatives for $W / W_{J}$. Finally, let $\lambda=x \varpi_{k} \in P$ be an arbitrary minuscule weight, where $x \in W^{J}$.

\subsubsection{Algebraic formula}

For $G$ simply laced, $\lambda$ as before and any $w \in W$, our first main result gives an algebraic expression for the product $e^{\lambda} \cdot\left[\mathcal{O}_{\mathbf{Q}_{G}(w)}\right] \in K_{H \times \mathbb{C}^{*}}\left(\mathbf{Q}_{G}\right)$ in terms of the right $q$-Heisenberg action on $K_{H \times \mathbb{C}^{*}}\left(\mathbf{Q}_{G}^{\text {rat }}\right)$. This is given explicitly as a sum over a set $\mathbf{Q} \mathbf{W}_{\lambda, w}$ of walks $\left(w_{1}, \ldots, w_{n}\right)$ in the quantum Bruhat graph QBG $(W)$ [3], beginning at $w_{0}=w$ and with steps prescribed by a set of positive roots determined by $\lambda$. (Here $n$ is the length of the minimal representative of the coset $w_{\circ} W_{J}$.)

Theorem 1 (= Theorem 3.11). Assume that $G$ is of ADE type but not of type $E_{8}$. For any minuscule weight $\lambda=x \varpi_{k} \in P$, where $x \in W^{J}$, and any $w \in W$, we have

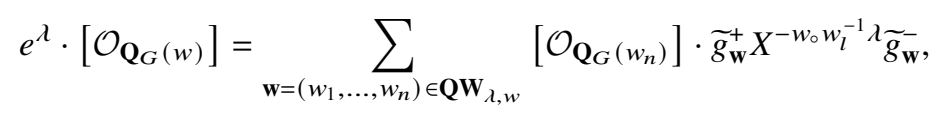

where $l=\ell(x)$ and $\widetilde{g}_{\mathbf{w}}^{+} X^{-w_{\circ} w_{l}^{-1} v \widetilde{g}_{\mathbf{w}}^{-}}$is an element of the q-Heisenberg algebra $\mathfrak{H}$ given explicitly by equations (3.63) and (3.64).

Acting by translations from the $q$-Heisenberg algebra, one immediately obtains a corresponding formula for $e^{\lambda} \cdot\left[\mathcal{O}_{\mathbf{Q}_{G}\left(w t_{\xi}\right)}\right] \in K_{H \times \mathbb{C}^{*}}\left(\mathbf{Q}_{G}^{\text {rat }}\right)$, for any $\xi \in Q^{\vee}$.

In order to prove Theorem 1, we apply the main result of [25]. The main step in our proof (see Theorem 3.7) is the intricate computation of a limit, as $t \rightarrow 0$, from the polynomial representation of the double affine Hecke algebra (DAHA). Thus one should also regard Theorem 1 as an explicit determination of all nonsymmetric $q$-Toda operators, in the sense of $[4,25]$, for minuscule weights in ADE type.

\subsubsection{Combinatorial formula}

Our second main result expresses the same product $e^{\lambda} \cdot\left[\mathcal{O}_{\mathbf{Q}_{G}(w)}\right]$ combinatorially. To achieve this, we enhance the set of quantum walks $\mathbf{Q} \mathbf{W}_{\lambda, w}$ to a set $\widetilde{\mathbf{Q W}}_{\lambda, w}$ of decorated quantum walks. Roughly speaking, a decorated quantum walk $(\mathbf{w}, \mathbf{b}) \in \widetilde{\mathbf{Q W}}_{\lambda, w}$ consists of a quantum walk $\mathbf{w}=\left(w_{1}, \ldots, w_{n}\right) \in$ $\mathbf{Q W} \mathbf{W}_{\lambda, w}$ together with a decoration $\mathbf{b}: S(\mathbf{w}) \rightarrow\{0,1\}$. The latter is a $\{0,1\}$-valued function on an explicit subset $S(\mathbf{w}) \subset\left\{t: w_{t}=w_{t-1}\right\}$ of the stationary steps in the walk w. Each $(\mathbf{w}, \mathbf{b}) \in \widetilde{\mathbf{Q W}}_{\lambda, w}$ carries a sign $(-1)^{(\mathbf{w}, \mathbf{b})} \in\{ \pm 1\}$, a weight $\operatorname{wt}(\mathbf{w}, \mathbf{b}) \in Q^{\vee}$ and a degree $\operatorname{deg}(\mathbf{w}, \mathbf{b}) \in \mathbb{Z}$. For details, see Section 3.4.1.

Our combinatorial inverse Chevalley formula reads as follows:

Theorem 2 (= Theorem 3.14). Assume that $G$ is of ADE type but not of type $E_{8}$. For any minuscule weight $\lambda=x \varpi_{k} \in P$, where $x \in W^{J}$, and any $w \in W$, we have

$$
\begin{aligned}
& e^{\lambda} \cdot\left[\mathcal{O}_{\mathbf{Q}_{G}(w)}\right] \\
& =\sum_{(\mathbf{w}, \mathbf{b}) \in \widehat{\mathbb{Q W}}_{\lambda, w}}(-1)^{(\mathbf{w}, \mathbf{b})} q^{\operatorname{deg}(\mathbf{w}, \mathbf{b})} \cdot\left[\mathcal{O}_{\mathbf{Q}_{G}\left(w_{n} t_{-w_{\circ}(\mathbf{w t}(\mathbf{w}, \mathbf{b}))}\right)}\left(-w_{\circ} w_{l}^{-1} \lambda+\mathrm{wt}(\mathbf{w}, \mathbf{b})\right)\right] .
\end{aligned}
$$

Theorem 2 is obtained as an immediate consequence of Theorem 1, by fully expanding the right-hand side of equation (1.5) in the $q$-Heisenberg algebra. Our combinatorial framework is designed to record 
the terms in this expansion. We find it satisfying that the DAHA-based limit used to prove equation (1.5) automatically manufactures the combinatorics of decorated quantum walks necessary for both formulas. We mention that equation (1.5) gives the extension to $K_{H \times \mathbb{C}^{*}}\left(\mathbf{Q}_{G}\right)$ of Lenart's rule [17, Theorem 3.1], which holds in $K(S L(n+1) / B)$, for multiplying a Grothendieck polynomial by a variable.

In Appendix A we work out some further details in the type $A$ case, including concrete examples of equation (1.5) in equations (A.1) and (A.2) and the important special case $w=w_{\circ}, k=1$ in equation (A.7). We also explain, following [25, §5.1], how to obtain the usual $q$-Toda difference operator by symmetrising Theorem 1.

\subsection{The sequel}

In [16] we will establish a different, equivalent version of our inverse Chevalley formula (1.6) in terms of paths (instead of walks) in the quantum Bruhat graph. By means of this alternate formula, we will give a separate and logically independent proof of Theorem 2 in type $A$, based on the Chevalley formulas of $[13,24]$ and an equivalent set of character identities for Demazure submodules of level 0 extremal weight modules. Finally, we will use Theorem 2 to derive a corresponding inverse Chevalley formula in the quantum $K$-ring $Q K_{H}(G / B)$, by means of Kato's isomorphism.

\section{Basic notation}

\subsection{Root system}

Let $G$ be a simply connected simple algebraic group over $\mathbb{C}$. As in the introduction, we fix a maximal torus and Borel subgroup $H \subset B \subset G$. Set $\mathfrak{g}:=\operatorname{Lie}(G)$ and $\mathfrak{h}:=\operatorname{Lie}(H)$. We denote by $\langle\cdot, \cdot\rangle: \mathfrak{h}^{*} \times \mathfrak{h} \rightarrow \mathbb{C}$ the canonical pairing, where $\mathfrak{h}^{*}=\operatorname{Hom}_{\mathbb{C}}(\mathfrak{h}, \mathbb{C})$.

Let $\Delta \subset \mathfrak{h}^{*}$ be the root system of $\mathfrak{g}, \Delta^{+} \subset \Delta$ the positive roots (with respect to $B$ ) and $\left\{\alpha_{i}\right\}_{i \in I} \subset \Delta^{+}$ the set of simple roots. We denote by $\alpha^{\vee} \in \mathfrak{h}$ the coroot corresponding to $\alpha \in \Delta$. Also, we denote by $\theta \in \Delta^{+}$the highest root of $\Delta$, and we set $\rho:=(1 / 2) \sum_{\alpha \in \Delta^{+}} \alpha$. The root lattice $Q$ and the coroot lattice $Q^{\vee}$ of $\mathfrak{g}$ are $Q:=\sum_{i \in I} \mathbb{Z} \alpha_{i}$ and $Q^{\vee}:=\sum_{i \in I} \mathbb{Z} \alpha_{i}^{\vee}$.

For $i \in I$, let $\varpi_{i} \in \mathfrak{h}^{*}$ be the fundamental weight determined by $\left\langle\varpi_{i}, \alpha_{j}^{\vee}\right\rangle=\delta_{i, j}$ for all $j \in I$, where $\delta_{i, j}$ denotes the Kronecker delta. The weight lattice $P$ of $\mathfrak{g}$ is defined by $P:=\sum_{i \in I} \mathbb{Z} \varpi_{i}$. We denote by $\mathbb{Z}[P]$ the group algebra of $P$ - that is, the associative algebra generated by formal elements $\left\{e^{\lambda} \mid \lambda \in P\right\}$, where the product is defined by $e^{\lambda} e^{\mu}:=e^{\lambda+\mu}$ for $\lambda, \mu \in P$.

A reflection $s_{\alpha} \in G L\left(\mathfrak{h}^{*}\right), \alpha \in \Delta$, is defined by $s_{\alpha}(\lambda):=\lambda-\left\langle\lambda, \alpha^{\vee}\right\rangle \alpha$ for $\lambda \in \mathfrak{h}^{*}$. We write $s_{i}:=s_{\alpha_{i}}$ for $i \in I$. Then the Weyl group $W:=\left\langle s_{i} \mid i \in I\right\rangle$ of $\mathfrak{g}$ is the subgroup of $G L\left(\mathfrak{h}^{*}\right)$ generated by $\left\{s_{i}\right\}_{i \in I}$. We denote by $\ell(w)$ the length of $w \in W$ with respect to $\left\{s_{i}\right\}_{i \in I}$.

\subsection{Quantum Bruhat graph}

The quantum Bruhat graph $\mathrm{QBG}(W)$ (compare [3, Definition 6.1]) is the $\Delta^{+}$-labelled directed graph whose vertices are the elements of $W$ and whose edges are of the form $x \stackrel{\alpha}{\rightarrow} y$, with $x, y \in W$ and $\alpha \in \Delta^{+}$, such that $y=x s_{\alpha}$ and either of the following holds: (B) $\ell(y)=\ell(x)+1$ or (Q) $\ell(y)=\ell(x)-2\left\langle\rho, \alpha^{\vee}\right\rangle+1$. An edge satisfying (B) (resp., (Q)) is called a Bruhat edge (resp., a quantum edge).

\subsection{Affine root system}

Let $\mathfrak{g}_{\mathrm{af}}:=\left(\mathfrak{g} \otimes \mathbb{C}\left[z, z^{-1}\right]\right) \oplus \mathbb{C} c \oplus \mathbb{C} d$ be the (untwisted) affine Lie algebra over $\mathbb{C}$ associated to $\mathfrak{g}$, where $c$ is the canonical central element and $d$ is the degree operator. Then $\mathfrak{h}_{\mathrm{af}}:=\mathfrak{h} \oplus \mathbb{C} c \oplus \mathbb{C} d$ is the Cartan subalgebra of $\mathfrak{g}_{\text {af }}$. We denote by $\langle\cdot, \cdot\rangle: \mathfrak{h}_{\text {af }}^{*} \times \mathfrak{h}_{\text {af }} \rightarrow \mathbb{C}$ the canonical pairing. Regarding $\lambda \in \mathfrak{h}^{*}$ as $\lambda \in \mathfrak{h}_{\mathrm{af}}^{*}=\operatorname{Hom}_{\mathbb{C}}\left(\mathfrak{h}_{\mathrm{af}}, \mathbb{C}\right)$ by setting $\langle\lambda, c\rangle=\langle\lambda, d\rangle=0$, we have $\mathfrak{h}^{*} \subset \mathfrak{h}_{\mathrm{af}}^{*}$. In this identification, we see that the canonical pairing $\langle\cdot, \cdot\rangle$ on $\mathfrak{h}_{\text {af }}^{*} \times \mathfrak{h}_{\text {af }}$ extends that on $\mathfrak{h}^{*} \times \mathfrak{h}$. 
Let us consider the root system of $\mathfrak{g}_{\mathrm{af}}$. We define $\delta$ to be the unique element of $\mathfrak{h}_{\mathrm{af}}^{*}$ which satisfies $\langle\delta, h\rangle=0$ for all $h \in \mathfrak{h},\langle\delta, c\rangle=0$ and $\langle\delta, d\rangle=1$. We set $\alpha_{0}:=-\theta+\delta \in \mathfrak{h}_{\mathrm{af}}^{*}$. Then the root system $\Delta_{\mathrm{af}}$ of $\mathfrak{g}_{\text {af }}$ has simple roots $\left\{\alpha_{i}\right\}_{i \in I_{\mathrm{af}}}$, where $I_{\mathrm{af}}:=I \sqcup\{0\}$.

For each $\alpha \in \Delta_{\text {af }}$, we have a reflection $s_{\alpha} \in G L\left(\mathfrak{h}_{\mathrm{af}}\right)$, defined as for $\mathfrak{g}$. Note that for $\alpha \in \Delta \subset \Delta_{\mathrm{af}}$, the restriction of a reflection $s_{\alpha}$ defined on $\mathfrak{h}_{\text {af }}$ to $\mathfrak{h}$ coincides with a reflection $s_{\alpha}$ defined on $\mathfrak{h}$. Set $s_{i}:=s_{\alpha_{i}}$ for $i \in I_{\text {af }}$. Then the Weyl group $W_{\text {af }}$ of $\mathfrak{g}_{\text {af }}$ (called the affine Weyl group) is defined to be the subgroup of $G L\left(\mathfrak{h}_{\mathrm{af}}\right)$ generated by $\left\{s_{i}\right\}_{i \in I_{\mathrm{af}}}$, namely $W_{\mathrm{af}}=\left\langle s_{i} \mid i \in I_{\mathrm{af}}\right\rangle$. In [9, §6.5], it is shown that $W_{\text {af }} \simeq W \ltimes\left\{t_{\alpha^{\vee}} \mid \alpha^{\vee} \in Q^{\vee}\right\} \simeq W \ltimes Q^{\vee}$, where $t_{\alpha^{\vee}}$ is the translation element corresponding to $\alpha^{\vee} \in Q^{\vee}$.

\subsection{Simply laced assumption}

While the definitions we have given are completely general, we will in fact assume throughout that $G$ is simply laced. As a result, we almost always identify $\mathfrak{h}^{*}$ with $\mathfrak{h}$, roots with coroots and so on. We do this by means of the nondegenerate $W$-invariant symmetric bilinear form $(\cdot, \cdot): \mathfrak{h}^{*} \times \mathfrak{h}^{*} \rightarrow \mathbb{C}$, normalised so that $(\alpha, \alpha)=2$ for all $\alpha \in \Delta$. We write $|\lambda|^{2}=(\lambda, \lambda)$ for $\lambda \in \mathfrak{h}^{*}$.

\subsection{Extended affine Weyl group}

Let $W_{\mathrm{ex}}=W \ltimes P$ be the extended affine Weyl group, which we let act on $P \oplus \mathbb{Z} \frac{\delta}{e}$ by the level 0 action

$$
w t_{\mu}(\lambda)=w(\lambda)-(\mu, \lambda) \delta, \quad w t_{\mu}(\delta)=\delta .
$$

Here we choose $e$ to be the smallest positive integer such that $e \cdot(P, P) \subset \mathbb{Z}$.

We also define the group $\Pi=P / Q$, which we realise as the subgroup of length 0 elements in $W_{\mathrm{ex}}$.

\subsection{Parameters}

Let us introduce a parameter $q^{1 / e}$ such that $\left(q^{1 / e}\right)^{e}=q$, where $q \in R\left(\mathbb{C}^{*}\right)$ is the equivariant parameter corresponding to loop rotation on $\mathbf{Q}_{G}^{\text {rat }}$. We will also use a related, but distinct parameter $q^{1 / e}$, as well as parameters $t, v$ such that $t=v^{2}$. Our base field for DAHA constructions will be $K=\mathbb{Q}\left(q^{1 / e}, v\right)$.

\subsection{Matrices}

For any ring $R$ with 1 , let $\operatorname{Mat}_{W}(R)$ denote the $R$-algebra of $W \times W$ matrices with entries in $R$. Let $\left\{e_{w}\right\}_{w \in W}$ be the standard basis of the free $R$-module $R^{|W|}$. For $A \in \operatorname{Mat}_{W}(R)$, we denote by $A_{w, \bullet}=\sum_{v \in W} A_{w, v} e_{v}$ the row of the matrix $A$ indexed by $w \in W$.

\section{Inverse Chevalley formula via DAHA}

In this section we use the methods of [25], based on the $\left(\mathbb{H}_{0}, \mathfrak{H}\right)$-bimodule structure of $K_{H \times \mathbb{C}^{*}}\left(\mathbf{Q}_{G}^{\text {rat }}\right)$, to prove the inverse Chevalley formulas (1.5) and (1.6).

\subsection{K-groups}

Let $K_{\tilde{\mathbf{I}}}\left(\mathbf{Q}_{G}^{\text {rat }}\right)$ be the equivariant $K$-group of $\mathbf{Q}_{G}^{\text {rat }}$ introduced in $[13, \S 6]$, where $\tilde{\mathbf{I}}=\mathbf{I} \rtimes \mathbb{C}^{*}$ is a semidirect product of the Iwahori subgroup $\mathbf{I} \subset G(\mathbb{C}[[z]])$ and loop rotation $\mathbb{C}^{*}$. Correspondingly, $K_{\tilde{\mathbf{I}}}\left(\mathbf{Q}_{G}^{\text {rat }}\right)$ is a module over $\mathbb{Z}[P]\left(\left(q^{-1}\right)\right)$, which acts by equivariant scalar multiplication.

One has the following classes in $K_{\tilde{\mathbf{I}}}\left(\mathbf{Q}_{G}^{\text {rat }}\right)$, for each $x \in W_{\text {af }}$ and $\lambda \in P$ :

- Schubert classes $\left[\mathcal{O}_{\mathbf{Q}_{G}(x)}\right]$

- Equivariant line bundle classes $[\mathcal{O}(\lambda)]$

○ Classes $\left[\mathcal{O}_{\mathbf{Q}_{G}(x)}(\lambda)\right]$ corresponding to the tensor product sheaves $\mathcal{O}_{\mathbf{Q}_{G}(x)} \otimes \mathcal{O}(\lambda)$. 
We follow the conventions of [13] for indexing equivariant line bundles and Schubert varieties in $\mathbf{Q}_{G}^{\text {rat }}$.

Definition 3.1 $\left(\left(H \times \mathbb{C}^{*}\right)\right.$-equivariant $K$-groups of $\mathbf{Q}_{G}^{\text {rat }}$ and $\left.\mathbf{Q}_{G}\right)$. Let $K_{H \times \mathbb{C}^{*}}\left(\mathbf{Q}_{G}^{\text {rat }}\right)$ be the $\mathbb{Z}\left[q^{ \pm 1}\right][P]$ submodule of $K_{\tilde{\mathbf{I}}}\left(\mathbf{Q}_{G}^{\text {rat }}\right)$ consisting of all convergent infinite $\mathbb{Z}\left[q^{ \pm 1}\right][P]$-linear combinations of Schubert classes $\left[\mathcal{O}_{\mathbf{Q}_{G}(x)}\right]$ for $x \in W_{\mathrm{af}}$, where convergence holds in the sense of [13, Proposition 5.11].

Similarly, we define $K_{H \times \mathbb{C}^{*}}\left(\mathbf{Q}_{G}\right)$ to be the $\mathbb{Z}\left[q^{ \pm 1}\right][P]$-submodule of $K_{\tilde{\mathbf{I}}}\left(\mathbf{Q}_{G}^{\text {rat }}\right)$ consisting of all convergent infinite $\mathbb{Z}\left[q^{ \pm 1}\right][P]$-linear combinations of Schubert classes $\left[\mathcal{O}_{\mathbf{Q}_{G}(x)}\right]$ for $x \in W_{\mathrm{af}}^{\geq 0}$.

The classes $\left\{\left[\mathcal{O}_{\mathbf{Q}_{G}(x)}\right]\right\}_{x \in W_{\text {af }}}$ satisfy a notion of topological linear independence in $K_{\tilde{\mathbf{I}}}\left(\mathbf{Q}_{G}^{\text {rat }}\right)$ given by [13, Proposition 5.11]; thus they form a topological $\mathbb{Z}\left[q^{ \pm 1}\right][P]$-basis of $K_{H \times \mathbb{C}^{*}}\left(\mathbf{Q}_{G}^{\text {rat }}\right)$. Also, one has $\left[\mathcal{O}_{\mathbf{Q}_{G}(x)}(\lambda)\right] \in K_{H \times \mathbb{C}^{*}}\left(\mathbf{Q}_{G}^{\text {rat }}\right)$ for any $x \in W_{\text {af }}$ and $\lambda \in P$, thanks to the two Chevalley formulas for dominant weights [13] and antidominant weights [24]. Similar (in fact, equivalent) assertions hold for $K_{H \times \mathbb{C}^{*}}\left(\mathbf{Q}_{G}\right)$.

Definition 3.2. Define $\mathbb{K} \subset K_{H \times \mathbb{C}^{*}}\left(\mathbf{Q}_{G}^{\text {rat }}\right)$ to be the $\mathbb{Z}\left[q^{ \pm 1}\right]$-submodule consisting of all finite $\mathbb{Z}\left[q^{ \pm 1}\right]$ linear combinations of the classes $\left\{\left[\mathcal{O}_{\mathbf{Q}_{G}(x)}(\lambda)\right]\right\}_{x \in W_{\mathrm{af}}, \lambda \in P}$.

By definition, $\mathbb{K}$ is only a $\mathbb{Z}\left[q^{ \pm 1}\right]$-submodule of $K_{H \times \mathbb{C}^{*}}\left(\mathbf{Q}_{G}^{\text {rat }}\right)$. But, as shown in [25, Theorem 5.1], it is indeed a $\mathbb{Z}\left[q^{ \pm 1}\right][P]$-submodule of $K_{H \times \mathbb{C}^{*}}\left(\mathbf{Q}_{G}^{\text {rat }}\right)$. We note that the classes $\left\{\left[\mathcal{O}_{\mathbf{Q}_{G}(x)}(\lambda)\right]\right\}_{x \in W_{\mathrm{af}}, \lambda \in P}$ are linearly independent over $\mathbb{Z}\left[q^{ \pm 1}\right]$, by the Chevalley formula of [13].

To summarise, we have the following chain of $\mathbb{Z}\left[q^{ \pm 1}\right][P]$-modules, and $K_{\tilde{\mathbf{I}}}\left(\mathbf{Q}_{G}^{\text {rat }}\right)$ is in fact a $\mathbb{Z}[P]\left(\left(q^{-1}\right)\right)$-module:

$$
\mathbb{K} \subset K_{H \times \mathbb{C}^{*}}\left(\mathbf{Q}_{G}^{\text {rat }}\right) \subset K_{\tilde{\mathbf{I}}}\left(\mathbf{Q}_{G}^{\mathrm{rat}}\right),
$$

where $\mathbb{K}$ is equipped with the $\mathbb{Z}\left[q^{ \pm 1}\right]$-basis $\left\{\left[\mathcal{O}_{\mathbf{Q}_{G}(x)}(\lambda)\right]\right\}_{x \in W_{\mathrm{af}}, \lambda \in P}$, and $K_{H \times \mathbb{C}^{*}}\left(\mathbf{Q}_{G}^{\mathrm{rat}}\right)$ with the topo$\operatorname{logical} \mathbb{Z}\left[q^{ \pm 1}\right][P]$-basis $\left\{\left[\mathcal{O}_{\mathbf{Q}_{G}(x)}\right]\right\}_{x \in W_{\text {af }}}$. Next we discuss additional structures on these modules.

\subsection{1. $q$-Heisenberg algebra}

Let $\widehat{\mathfrak{H}}$ be the $q$-Heisenberg algebra defined as a $\mathbb{Z}\left[q^{ \pm 1 / e}\right]$-algebra by generators

$$
X^{v}(v \in P), \quad t_{\mu}(\mu \in P),
$$

and relations

$$
\begin{gathered}
X^{\lambda} X^{v}=X^{\lambda+v} \\
t_{\mu} t_{\xi}=t_{\mu+\xi} \\
X^{0}=t_{0}=1 \\
X^{v} t_{\mu}=q^{(\mu, v)} t_{\mu} X^{v}
\end{gathered}
$$

for all $\lambda, \mu, v, \xi \in P$.

Let $\mathfrak{H} \subset \widehat{\mathfrak{H}}$ be the $\mathbb{Z}\left[q^{ \pm 1}\right]$-subalgebra generated by $X^{v}(\nu \in P)$ and $t_{\beta}(\beta \in Q)$.

Proposition 3.3. There exists a unique right $\mathfrak{H}$-module structure on $\mathbb{K}$ and $K_{H \times \mathbb{C}^{*}}\left(\mathbf{Q}_{G}^{\text {rat }}\right)$ such that

$$
\begin{gathered}
{\left[\mathcal{O}_{\mathbf{Q}_{G}(x)}(\lambda)\right] \cdot X^{v}=\left[\mathcal{O}_{\mathbf{Q}_{G}(x)}(\lambda+v)\right]} \\
{\left[\mathcal{O}_{\mathbf{Q}_{G}(x)}(\lambda)\right] \cdot t_{\beta}=q^{(\beta, \lambda)} \cdot\left[\mathcal{O}_{\mathbf{Q}_{G}\left(x t_{-w_{\circ} \beta}\right)}(\lambda)\right]}
\end{gathered}
$$


for all $x \in W_{\mathrm{af}}, \lambda, v \in P$ and $\beta \in Q$. Moreover, $\mathbb{K}$ is free as an $\mathfrak{H}$-module, with $\mathfrak{H}$-basis given by $\left\{\left[\mathcal{O}_{\mathbf{Q}_{G}(w)}\right]\right\}_{w \in W}$.

Proof. See [10] and [25, Proposition 2.3]. We note that our conventions here differ slightly from those of [25], where the class $\left[\mathcal{O}_{\mathbf{Q}_{G}(x)}\right]$ is denoted by $\left[\overline{\mathcal{O}}_{x}\right]$. This accounts for the twist by $-w_{\circ}$ in the action of $t_{\beta}$.

\subsubsection{Nil-DAHA}

Next we turn to the nil-DAHA $\mathbb{H}_{0}$, which is the $\mathbb{Z}\left[\mathrm{q}^{ \pm 1}\right]$-algebra (note the notational distinction between $q$ and $q$ ) defined by generators

$$
T_{i}\left(i \in I_{\mathrm{af}}\right), \quad X^{v}(v \in P),
$$

and relations

$$
\begin{gathered}
T_{i} T_{j} \cdots=T_{j} T_{i} \cdots \quad\left(m_{i j}=\left|s_{i} s_{j}\right| \text { factors on both sides }\right) \\
T_{i}\left(T_{i}+1\right)=0 \\
X^{0}=1 \\
X^{v} X^{\mu}=X^{v+\mu} \\
T_{i} X^{v}=X^{s_{i}(v)} T_{i}-\frac{X^{v}-X^{s_{i}(v)}}{1-X^{\alpha_{i}}}
\end{gathered}
$$

where it is understood that $X^{\delta}=\mathrm{q}$. We also use $D_{i}=1+T_{i}\left(i \in I_{\mathrm{af}}\right)$. These elements satisfy the braid relations as before and quadratic relations $D_{i}^{2}=D_{i}$.

Proposition 3.4 [13, Theorem 6.5]. There is a left $\mathbb{H}_{0}$-action on $K_{H \times \mathbb{C}^{*}}\left(\mathbf{Q}_{G}^{\text {rat }}\right)$ uniquely determined by

$$
\begin{gathered}
\mathbf{q} \cdot\left[\mathcal{O}_{\mathbf{Q}_{G}\left(t_{\alpha}\right)}(\lambda)\right]=q^{-1}\left[\mathcal{O}_{\mathbf{Q}_{G}\left(t_{\alpha}\right)}(\lambda)\right] \\
X^{v} \cdot\left[\mathcal{O}_{\mathbf{Q}_{G}\left(t_{\alpha}\right)}(\lambda)\right]=e^{-v}\left[\mathcal{O}_{\mathbf{Q}_{G}\left(t_{\alpha}\right)}(\lambda)\right] \\
D_{i} \cdot\left[\mathcal{O}_{\mathbf{Q}_{G}\left(t_{\alpha}\right)}(\lambda)\right]=\left[\mathcal{O}_{\mathbf{Q}_{G}\left(t_{\alpha}\right)}(\lambda)\right] \\
D_{0} \cdot\left[\mathcal{O}_{\mathbf{Q}_{G}\left(t_{\alpha}\right)}(\lambda)\right]=\left[\mathcal{O}_{\mathbf{Q}_{G}\left(s_{0} t_{\alpha}\right)}(\lambda)\right]
\end{gathered}
$$

for all $i \in I, \alpha \in Q$ and $v, \lambda \in P$.

We warn the reader that $X^{v}(v \in P)$ can be viewed both as an element of $\mathbb{H}_{0}$ and as an element of $\mathfrak{h}$. Its left and right actions on $K_{H \times \mathbb{C}^{*}}\left(\mathbf{Q}_{G}^{\text {rat }}\right)$ differ drastically. From the left, $X^{v} \in \mathbb{H}_{0}$ acts as equivariant scalar multiplication by $e^{-v}$, whereas from the right, $X^{v} \in \mathfrak{S}$ acts as the line bundle twist by $\mathcal{O}(v)$.

\subsection{3. $\mathbb{K}$ as a bimodule}

By [25, Theorem 5.1], the $\mathbb{H}_{0}$-action on $K_{H \times \mathbb{C}^{*}}\left(\mathbf{Q}_{G}^{\text {rat }}\right)$ leaves $\mathbb{K}$ stable. Thus $\mathbb{K}$ is an $\left(\mathbb{H}_{0}, \mathfrak{H}\right)$-bimodule. Since $\mathbb{K}$ is free as a right $\mathfrak{G}$-module, the nil-DAHA action on $\mathbb{K}$ and hence on $K_{H \times \mathbb{C}^{*}}\left(\mathbf{Q}_{G}^{\text {rat }}\right)$ is therefore characterised by the unique ring homomorphism $\varrho_{0}: \mathbb{H}_{0} \rightarrow$ Mat ${ }_{W}(\mathfrak{G})$ satisfying

$$
H \cdot\left[\mathcal{O}_{\mathbf{Q}_{G}(w)}\right]=\sum_{v \in W}\left[\mathcal{O}_{\mathbf{Q}_{G}(v)}\right] \cdot \varrho_{0}(H)_{v w}
$$

for all $H \in \mathbb{H}_{0}$ and $w \in W$. 
For us, the key point is that the matrices encoding the inverse Chevalley formula (1.2) are exactly $\varrho_{0}\left(X^{-\lambda}\right)$ for $\lambda \in P$.

\subsubsection{Further observations}

For any $i \in I_{\mathrm{af}}$ and $x \in W_{\mathrm{af}}$, we have

$$
D_{i} \cdot\left[\mathcal{O}_{\mathbf{Q}_{G}(x)}\right]= \begin{cases}{\left[\mathcal{O}_{\mathbf{Q}_{G}\left(s_{i} x\right)}\right]} & \text { if } s_{i} x<x, \\ {\left[\mathcal{O}_{\mathbf{Q}_{G}(x)}\right]} & \text { if } s_{i} x>x,\end{cases}
$$

where $\prec$ is the semi-infinite Bruhat order on $W_{\text {af }}$ (see [13] or [25]). In the special case where $i \in I$ and $x=w \in W$, we have

$$
D_{i} \cdot\left[\mathcal{O}_{\mathbf{Q}_{G}(w)}\right]= \begin{cases}{\left[\mathcal{O}_{\mathbf{Q}_{G}\left(s_{i} w\right)}\right]} & \text { if } s_{i} w<w, \\ {\left[\mathcal{O}_{\mathbf{Q}_{G}(w)}\right]} & \text { if } s_{i} w>w,\end{cases}
$$

where $<$ is the (usual) Bruhat order on $W$.

It follows from equation (3.20) and Proposition 3.4 (see also [13, Proposition 6.3]) that the nil affine Hecke $\mathbb{Z}\left[q^{ \pm 1}\right]$-subalgebra $\mathcal{H}_{0} \subset \mathbb{H}_{0}$ generated by

$$
D_{i}(i \in I), \quad X^{v}(v \in P),
$$

leaves $K_{H \times \mathbb{C}^{*}}\left(\mathbf{Q}_{G}\right) \subset K_{H \times \mathbb{C}^{*}}\left(\mathbf{Q}_{G}^{\text {rat }}\right)$ stable. (Our inverse Chevalley formulas make this explicit.)

We also deduce from equation (3.20) that $\left[\mathcal{O}_{\mathbf{Q}_{G}\left(w_{0}\right)}\right]$ generates $\mathbb{K}$ as an $\left(\mathcal{H}_{0}, \mathfrak{H}\right)$-bimodule (in fact, the action of $\mathfrak{S}$ and the $D_{i}$ for $i \in I$ on $\left[\mathcal{O}_{\mathbf{Q}_{G}\left(w_{\circ}\right)}\right]$ are sufficient to generate all of $\left.\mathbb{K}\right)$.

\subsection{DAHA}

The main result of [25] gives an algebraic construction of the homomorphism $\varrho_{0}: \mathbb{H}_{0} \rightarrow \operatorname{Mat}_{W}(\mathfrak{H})$ of equation (3.18), as a limit as $\mathrm{t} \rightarrow 0$ from the polynomial representation of DAHA. Let us recall the necessary details of this construction.

\subsubsection{Definition of DAHA}

Recall that $\mathrm{K}=\mathbb{Q}\left(q^{1 / e}, \mathrm{v}\right)$, where $\mathrm{t}=\mathrm{v}^{2}$. The (extended) DAHA $\widehat{\mathbb{H}}$ is the $\mathrm{K}$-algebra defined by generators

$$
T_{i}\left(i \in I_{\mathrm{af}}\right), \quad X^{v}(v \in P), \quad \pi(\pi \in \Pi),
$$

and relations

$$
\begin{gathered}
T_{i} T_{j} \cdots=T_{j} T_{i} \cdots \quad\left(m_{i j}=\left|s_{i} s_{j}\right| \text { factors on both sides }\right) \\
\left(T_{i}-\mathrm{t}\right)\left(T_{i}+1\right)=0 \\
X^{v} X^{\mu}=X^{v+\mu} \\
X^{0}=1 \\
T_{i} X^{v}=X^{s_{i}(v)} T_{i}+(\mathrm{t}-1)\left(1-X^{\alpha_{i}}\right)^{-1}\left(X^{v}-X^{s_{i}(v)}\right) \\
\pi T_{i} \pi^{-1}=T_{j} \quad\left(\pi\left(\alpha_{i}\right)=\alpha_{j}\right) \\
\pi X^{v} \pi^{-1}=X^{\pi(v)}
\end{gathered}
$$

for all $i, j \in I_{\text {af }}, v, \mu \in P$ and $\pi \in \Pi$. Here it is understood that $X^{\delta / e}=\mathrm{q}^{1 / e}$. 
One has well-defined elements $Y^{v} \in \widehat{\mathbb{H}}(v \in P)$ given by

$$
Y^{v}=\mathrm{v}^{-\sum_{k=1}^{\ell} \epsilon_{k}} \pi T_{i_{1}}^{\epsilon_{1}} \cdots T_{i_{\ell}}^{\epsilon_{\ell}}
$$

for any reduced expression $t_{v}=\pi s_{i_{1}} \cdots s_{i_{\ell}} \in P \rtimes W=W_{\mathrm{ex}}$, where $i_{k} \in I_{\mathrm{af}}$ and $\pi \in \Pi$, and where $\epsilon_{k} \in\{ \pm 1\}$ are determined by

$$
\epsilon_{k}= \begin{cases}+1 & \text { if } \pi s_{i_{1}} \cdots s_{i_{k-1}}\left(\alpha_{i_{k}}\right) \in-\Delta^{+}+\mathbb{Z} \delta \\ -1 & \text { if } \pi s_{i_{1}} \cdots s_{i_{k-1}}\left(\alpha_{i_{k}}\right) \in \Delta^{+}+\mathbb{Z} \delta\end{cases}
$$

These elements satisfy

$$
\begin{gathered}
Y^{v} Y^{\mu}=Y^{v+\mu} \\
Y^{0}=1 \\
T_{i} Y^{v}-Y^{s_{i}(v)} T_{i}=(\mathrm{t}-1)\left(1-Y^{-\alpha_{i}}\right)^{-1}\left(Y^{v}-Y^{s_{i}(v)}\right)
\end{gathered}
$$

for any $i \in I$ and $v, \mu \in P$. (To extend the latter relation to $i \in I_{\mathrm{af}}$, see, e.g., [25, §3.2].)

\subsubsection{Polynomial representation}

Let $\mathrm{K}(P)$ be the field of fractions of $\mathrm{K}[P]=\mathrm{K}\left[X^{v}: v \in P\right]$. The extended affine Weyl group $W_{\mathrm{ex}}$ acts on $\mathrm{K}[P]$ by automorphisms as follows: $t_{\mu} w\left(X^{v}\right)=q^{-(\mu, w v)} X^{w v}$. By a 'difference-reflection operator' we mean an element of the smash product

$$
\mathrm{K}(P) \rtimes W_{\mathrm{ex}} \cong \mathrm{K}(P) \otimes_{\mathrm{K}} \mathrm{K}\left[W_{\mathrm{ex}}\right] \quad \text { (K-linear isomorphism) }
$$

acting from the left on $\mathrm{K}(P)$ by multiplication operators (first tensor factor) and the induced action of $W_{\mathrm{ex}}$ (second tensor factor). We note that $\mathrm{K}[P] \rtimes P$, where $P \cong\left\{t_{\mu}: \mu \in P\right\} \subset W_{\mathrm{ex}}$, is nothing but the $q$-Heisenberg algebra $\widehat{\mathfrak{H}}$ with scalars extended to $\mathrm{K}$.

The polynomial representation $\varrho: \widehat{\mathbb{H}} \rightarrow \operatorname{End}(\mathrm{K}[P])$ is a faithful representation of $\widehat{\mathbb{H}}$ given as follows: $\varrho\left(X^{v}\right)$ is multiplication by $X^{v}$, the elements $\pi \in \Pi \subset W_{\text {ex }}$ act by the automorphisms already discussed and $T_{i}\left(i \in I_{\mathrm{af}}\right)$ act by Demazure-Lusztig operators:

$$
\varrho\left(T_{i}\right)=\frac{\mathrm{t} X^{\alpha_{i}}-1}{X^{\alpha_{i}}-1} s_{i}+\frac{1-\mathrm{t}}{X^{\alpha_{i}}-1} .
$$

In general, elements of $\widehat{\mathbb{H}}$ act on $\mathrm{K}[P]$ by difference-reflection operators from $\mathrm{K}(P) \rtimes W_{\mathrm{ex}}$; therefore, they are represented uniquely as sums of $f t_{\mu} u=f \otimes t_{\mu} u$, where $f \in \mathrm{K}(P)$ and $t_{\mu} u \in W_{\mathrm{ex}}$. Obviously, not all elements of $\mathrm{K}(P) \rtimes W_{\text {ex }}$ leave $\mathrm{K}[P]$ stable, but those from $\widehat{\mathbb{H}}$ do. As in equation (3.34), we will simultaneously regard $\varrho(H)$ for $H \in \widehat{\mathbb{H}}$ as an element of End $(\mathrm{K}[P])$ and as a difference-reflection operator - that is, an element of $\mathrm{K}(P) \rtimes W_{\text {ex }}$.

\subsubsection{Limiting procedure}

Consider the homomorphism

$$
\varkappa: \mathrm{K}(P) \rtimes W_{\mathrm{ex}} \rightarrow \mathrm{Mat}_{W}(\mathrm{~K}(P) \rtimes P),
$$

which for $f t_{\mu} u \in \mathrm{K}(P) \rtimes W_{\mathrm{ex}}$ and $v, w \in W$ is given by

$$
\varkappa\left(f t_{\mu} u\right)_{v w}= \begin{cases}\left.\left(v^{-1} \cdot f\right)\right|_{X^{v_{\mapsto}} \mathrm{t}^{-(\rho, \nu)} X^{\nu}} \mathrm{t}^{\left(\rho, v^{-1} \mu\right)} t_{v^{-1}(\mu)} & \text { if } v=u w \\ 0 & \text { otherwise. }\end{cases}
$$


By [25, Theorem 5.1], the sought-after matrices $\varrho_{0}\left(X^{-\lambda}\right)$ from equation (3.18) can be obtained by applying a simple automorphism to the result of the entry-wise limit

$$
\varrho_{0}^{\prime}\left(Y^{\lambda}\right):=\lim _{v \rightarrow 0} \varkappa\left(\varrho\left(Y^{\lambda}\right)\right) \in \operatorname{Mat}_{W}(\mathfrak{H}) .
$$

The existence of this limit for any $\lambda \in P$ is ensured by [4, Theorem 4.4]. The significance of $Y^{\lambda}$ here is that it is the image of $X^{-\lambda}$ under the DAHA duality anti-involution; see [25, §3.2] for further details.

\subsection{Computing limits}

Our goal is thus to compute certain $\varrho_{0}^{\prime}\left(Y^{\lambda}\right)$ given by formula (3.36). To do so, we will work with compositions of the auxiliary difference-reflection operators

$$
\mathrm{G}_{\eta}^{ \pm}=\frac{\mathrm{t}^{ \pm 1}-X^{\eta}}{1-X^{\eta}}+\frac{\mathrm{t}^{ \pm 1}-1}{1-X^{-\eta}} s_{\eta}
$$

for $\eta \in \Delta_{\text {af }}^{+}$. These are cousins of the Demazure-Lusztig operators $\varrho\left(T_{i}\right)$ :

$$
\mathrm{G}_{\alpha_{i}}^{+}=s_{i} \varrho\left(T_{i}\right), \quad \mathrm{G}_{\alpha_{i}}^{-}=\left(\mathrm{G}_{\alpha_{i}}^{+}\right)^{-1}=\varrho\left(T_{i}^{-1}\right) s_{i} .
$$

As we wish to take $\mathrm{v} \rightarrow 0$, all rational functions in such operators will be expanded in $\mathbb{Z}\left[\mathrm{q}^{ \pm 1 / e}\right][P]((\mathrm{v}))$. In this context, we shall write $A \approx B$ to mean that $A$ and $B$ have the same lowest term - that is, that $A$ and $B$ have the same order with respect to $v$ and that $A-B$ has strictly greater order than that of $A$ and $B$.

Set $\eta \in \Delta^{+}$. By equation (3.35), we see that a matrix entry of $\varkappa\left(\mathrm{G}_{\eta}^{ \pm}\right)$vanishes unless it is indexed by $(w, w)$ or $\left(w, s_{\eta} w\right)$ for some $w \in W$. The nonvanishing entries are given as follows:

$$
\begin{aligned}
& x\left(\mathrm{G}_{\eta}^{+}\right)_{w, w}=\frac{\mathrm{t}-\mathrm{t}^{-\left(\rho, w^{-1} \eta\right)} X^{w^{-1}(\eta)}}{1-\mathrm{t}^{-\left(\rho, w^{-1} \eta\right)} X^{w^{-1}(\eta)}} \approx \begin{cases}1 & \text { if } w^{-1} \eta>0, \\
\mathrm{t}\left(1-X^{w^{-1}} \eta\right) & \text { if }\left(\rho, w^{-1} \eta\right)=-1, \\
\mathrm{t} & \text { if }\left(\rho, w^{-1} \eta\right)<-1\end{cases} \\
& x\left(\mathrm{G}_{\eta}^{+}\right)_{w, s_{\eta} w}=\frac{\mathrm{t}-1}{1-\mathrm{t}^{\left(\rho, w^{-1} \eta\right)} X^{-w^{-1} \eta}} \approx \begin{cases}-1 & \text { if } w^{-1} \eta>0, \\
\mathrm{t}^{-\left(\rho, w^{-1} \eta\right)} X^{w^{-1} \eta} & \text { if } w^{-1} \eta<0\end{cases} \\
& x\left(\mathrm{G}_{\eta}^{-}\right)_{w, w}=\frac{\mathrm{t}^{-1}-\mathrm{t}^{-\left(\rho, w^{-1} \eta\right)} X^{w^{-1}(\eta)}}{1-\mathrm{t}^{-\left(\rho, w^{-1} \eta\right)} X^{w^{-1}(\eta)}} \approx \begin{cases}1-X^{-w^{-1} \eta} & \text { if }\left(\rho, w^{-1} \eta\right)=1, \\
1 & \text { if }\left(\rho, w^{-1} \eta\right)>1, \\
\mathrm{t}^{-1} & \text { if } w^{-1} \eta<0\end{cases} \\
& x\left(\mathrm{G}_{\eta}^{-}\right)_{w, s_{\eta} w}=\frac{\mathrm{t}^{-1}-1}{1-\mathrm{t}^{\left(\rho, w^{-1} \eta\right)} X^{-w^{-1} \eta}} \approx \begin{cases}\mathrm{t}^{-1} & \text { if } w^{-1} \eta>0, \\
-\mathrm{t}^{-\left(\rho, w^{-1} \eta\right)-1} X^{w^{-1} \eta} & \text { if } w^{-1} \eta<0 .\end{cases}
\end{aligned}
$$

Remark 3.5. These computations are closely related to those of [3, §4] involving quantum Bruhat operators. The precise connection to the quantum Bruhat graph will be made later.

\subsubsection{Expanding products as sums over walks}

Using the vanishing of the matrix coefficients, we obtain the following row expansion (see Section 2.7) for any $w \in W$ and any sequence $\vec{\eta}=\left(\eta_{1}, \ldots, \eta_{n}\right) \in\left(\Delta^{+}\right)^{n}$ :

$$
\varkappa\left(\mathrm{G}_{\eta_{1}}^{ \pm} \mathrm{G}_{\eta_{2}}^{ \pm} \cdots \mathrm{G}_{\eta_{n}}^{ \pm}\right)_{w, \bullet}=\sum_{\mathbf{w} \in \mathbf{W}_{w}^{\vec{\eta}}} \varkappa\left(\mathrm{G}_{\eta_{1}}^{ \pm}\right)_{w_{0}, w_{1}} \varkappa\left(\mathrm{G}_{\eta_{2}}^{ \pm}\right)_{w_{1}, w_{2}} \cdots \varkappa\left(\mathrm{G}_{\eta_{n}}^{ \pm}\right)_{w_{n-1}, w_{n}} e_{w_{n}},
$$


where $w_{0}=w$ and $\mathbf{W}_{w}^{\vec{\eta}}$ is the set of sequences $\mathbf{w}=\left(w_{1}, \ldots, w_{n}\right)$ of elements of $W$ such that

$$
w_{t} \in\left\{w_{t-1}, s_{\eta_{t}} w_{t-1}\right\} \text { for each } t=1, \ldots, n
$$

We call elements of $\mathbf{W}_{w}^{\vec{\eta}}$ walks in $W$.

\subsubsection{Quantum walks}

We will see that the matrices describing our inverse Chevalley formula are given by sums over subsets of walks given as follows:

Definition 3.6. We call $\mathbf{w}=\left(w_{1}, \ldots, w_{n}\right) \in \mathbf{W}_{w}^{\vec{\eta}}$ a quantum walk if it defines a directed walk in the quantum Bruhat graph QBG $(W)$ - that is, for each $1 \leq t \leq n$, either $w_{t}=w_{t-1}$ or $w_{t-1} \rightarrow w_{t}=s_{\eta_{t}} w_{t-1}$ is an edge in $\mathrm{QBG}(W)$.

We note that such an edge $w_{t-1} \rightarrow w_{t}$ in $\operatorname{QBG}(W)$ is labelled by $\pm w_{t-1}^{-1} \eta_{t}$. In the case when $w_{t-1}>w_{t}$, the requirement for an edge is that $\ell\left(w_{t-1}\right)-\ell\left(w_{t}\right)=\left(2 \rho,-w_{t-1}^{-1} \eta_{t}\right)-1$.

\subsubsection{Main limit}

Now we proceed to the limit computation which will give our first main result. Let $\varpi_{k} \in P_{+}$be a minuscule fundamental weight. (Thus we implicitly assume that our simply laced group $G$ is not of type $E_{8}$.) As in the introduction, let $J=I \backslash\{k\}$, let $W_{J}=\left\langle s_{j} \mid j \in J\right\rangle \subset W$ be the corresponding parabolic subgroup, which is the stabiliser of $\varpi_{k}$, and let $W^{J}$ denote the set of minimal coset representatives for $W / W_{J}$. For $w \in W$, let $\lfloor w\rfloor \in W^{J}$ be the minimal representative of its coset $w W_{J}$.

Fix $x \in W^{J}$ and let $\lambda=x \varpi_{k} \in P$, which is an arbitrary minuscule weight. Let $y \in W$ be the unique element such that $\left\lfloor w_{\circ}\right\rfloor=y x$. Since $\varpi_{k}$ is minuscule, we have $\ell\left(\left\lfloor w_{\circ}\right\rfloor\right)=\ell(y)+\ell(x)$; indeed, on $W^{J}$, the Bruhat order coincides with the left weak Bruhat order [6, Lemma 11.1.16].

We fix reduced expressions $x=s_{j_{l}} \cdots s_{j_{1}}$ and $y=s_{i_{1}} \cdots s_{i_{m}}$, and we define

$$
\begin{array}{cl}
\beta_{r}=s_{j_{l}} s_{j_{l-1}} \cdots s_{j_{r+1}}\left(\alpha_{j_{r}}\right), & 1 \leq r \leq l, \\
\gamma_{s}=s_{i_{m}} s_{i_{m-1}} \cdots s_{i_{s+1}}\left(\alpha_{i_{s}}\right), & 1 \leq s \leq m .
\end{array}
$$

Hence, if we set

$$
\operatorname{Inv}(w)=\Delta^{+} \cap w\left(-\Delta^{+}\right)
$$

then $\operatorname{Inv}(x)=\left\{\beta_{1}, \ldots, \beta_{l}\right\}$ and $\operatorname{Inv}\left(y^{-1}\right)=\left\{\gamma_{1}, \ldots, \gamma_{m}\right\}$.

Now, for any $w \in W$, define the set of walks $\mathbf{W}_{\lambda, w}=\mathbf{W}_{w}^{\vec{\eta}}$, where $\vec{\eta}$ is given by

$$
\vec{\eta}=\left(\eta_{1}, \ldots, \eta_{n}\right)=\left(\beta_{l}, \ldots, \beta_{1}, \gamma_{1}, \ldots, \gamma_{m}\right)
$$

and $n=l+m$. Let $\mathbf{Q} \mathbf{W}_{\lambda, w}$ denote the subset of quantum walks in $\mathbf{W}_{w}^{\vec{\eta}}$. (These sets depend on the choice of reduced expressions for $x$ and $y$, even though our notation does not indicate this.)

The inverse Chevalley formula for minuscule weights is an immediate consequence of the following, which is the main technical achievement of this paper:

Theorem 3.7. For any minuscule $\lambda \in P$ and $w \in W$, we have

$$
\varrho_{0}^{\prime}\left(Y^{\lambda}\right)_{w, \bullet}=\sum_{\mathbf{w}=\left(w_{1}, \ldots, w_{n}\right) \in \mathbf{Q} \mathbf{W}_{\lambda, w}} g_{\mathbf{w}}^{-} t_{w_{l}^{-1} \lambda} g_{\mathbf{w}}^{+} e_{w_{n}}
$$


with

$$
\begin{aligned}
& g_{\mathbf{w}}^{-}=\prod_{1 \leq t \leq l} \begin{cases}1-X^{-w_{t-1}^{-1}} \eta_{t} & \text { if } w_{t}=w_{t-1} \text { and }\left(\rho, w_{t-1}^{-1} \eta_{t}\right)=1, \\
-X^{w_{t-1}^{-1} \eta_{t}} & \begin{array}{l}
\text { if } w_{t}<w_{t-1}, \\
\text { otherwise }
\end{array}\end{cases} \\
& g_{\mathbf{w}}^{+}=\prod_{l<t \leq n} \begin{cases}1-X^{w_{t-1}^{-1} \eta_{t}} & \text { if } w_{t}=w_{t-1} \text { and }\left(\rho, w_{t-1}^{-1} \eta_{t}\right)=-1, \\
X^{w_{t-1}^{-1} \eta_{t}} & \text { if } w_{t}<w_{t-1}, \\
-1 & \text { if } w_{t}>w_{t-1}, \\
1 & \text { otherwise. }\end{cases}
\end{aligned}
$$

For the proof of Theorem 3.7, we will need the following notions, which rely heavily on $\lambda \in P$ being minuscule. For $w \in W$, and with all other notation as before, let us define

$$
\begin{array}{cc}
\operatorname{Inv}(w)_{\lambda}^{+}=\{\gamma \in \operatorname{Inv}(w) \mid(\gamma, \lambda)=1\}, & \ell_{\lambda}^{+}(w)=\# \operatorname{Inv}(w)_{\lambda}^{+}, \\
\operatorname{Inv}(w)_{\lambda}^{-}=\{\gamma \in \operatorname{Inv}(w) \mid(\gamma, \lambda)=-1\}, & \ell_{\lambda}^{-}(w)=\# \operatorname{Inv}(w)_{\lambda}^{-},
\end{array}
$$

and

$$
\begin{array}{ll}
\operatorname{Inv}(w)_{\lambda, r}^{-}=\operatorname{Inv}(w) \cap\left\{\beta_{r}, \beta_{r+1}, \ldots, \beta_{l}\right\}, & \ell_{\lambda, r}^{-}(w)=\# \operatorname{Inv}(w)_{\lambda, r}^{-}, \\
\operatorname{Inv}(w)_{\lambda, s}^{+}=\operatorname{Inv}(w) \cap\left\{\gamma_{s}, \gamma_{s+1}, \ldots, \gamma_{m}\right\}, & \ell_{\lambda, s}^{+}(w)=\# \operatorname{Inv}(w)_{\lambda, s}^{+},
\end{array}
$$

where $1 \leq r \leq l$ and $1 \leq s \leq m$.

The following lemma seems to be well known, but we give a simple proof in Appendix B for the convenience of the reader:

Lemma 3.8. With all notation as before, the following are true:

1. We have

2. For any $w \in W$,

$$
2 \ell(x)-\ell\left(\left\lfloor w_{\circ}\right\rfloor\right)=-2(\rho, \lambda) .
$$

and

$$
(\rho-w \rho, \lambda)=\ell_{\lambda}^{+}(w)-\ell_{\lambda}^{-}(w)
$$

$$
\begin{gathered}
\operatorname{Inv}(w)_{\lambda}^{-}=\operatorname{Inv}(w) \cap \operatorname{Inv}(x), \\
\operatorname{Inv}(w)_{\lambda}^{+}=\operatorname{Inv}(w) \cap \operatorname{Inv}\left(y^{-1}\right) .
\end{gathered}
$$

The proof of the following proposition will be given in Appendix B:

Proposition 3.9. With all notation as before, the following are true:

1. If $s_{\beta_{r}} w<w$ for $1 \leq r \leq l$, then

$$
\ell(w)-\ell\left(s_{\beta_{r}} w\right)=2\left(\ell_{\lambda, r}^{-}(w)-\ell_{\lambda, r}^{-}\left(s_{\beta_{r}} w\right)\right)-1 .
$$

2. If $s_{\gamma_{s}} w<w$ for $1 \leq s \leq m$, then

$$
\ell(w)-\ell\left(s_{\gamma_{s}} w\right)=2\left(\ell_{\lambda, s}^{+}(w)-\ell_{\lambda, s}^{+}\left(s_{\gamma_{s}} w\right)\right)-1 .
$$

Remark 3.10. Equations (3.59) and (3.60) generalise the following well-known formula for length differences in the symmetric group $S_{n}$ :

$$
\ell(w)-\ell((i j) w)=1+2 \cdot \mid\left\{k: i<k<j \text { and } w^{-1}(i)>w^{-1}(k)>w^{-1}(j)\right\} \mid,
$$


where $1 \leq i<j \leq n, w \in S_{n}$ is a permutation such that $w^{-1}(i)>w^{-1}(j)$, and $(i j)$ denotes the transposition swapping $i$ and $j$.

Proof of Theorem 3.7. Let $\pi \in \Pi$ be the length 0 element of $W_{\mathrm{ex}}$ corresponding to coset $\varpi_{k}+Q$ in $P / Q$. We have the formula $t_{\varpi_{k}}=\pi\left\lfloor w_{\circ}\right\rfloor=\pi y x$ (which may be taken as the definition of $\pi$ ), and hence $t_{\lambda}=x \pi y$.

Using equations (3.33) and (3.55), we find

$$
\begin{aligned}
Y^{\lambda} & =\mathrm{v}^{2 \ell(x)-\ell\left(\left\lfloor w_{\circ}\right\rfloor\right)} T_{x^{-1}}^{-1} \pi T_{y} \\
& =\mathrm{t}^{-(\rho, \lambda)} T_{x^{-1}}^{-1} \pi T_{y}
\end{aligned}
$$

in $\widehat{\mathbb{H}}$, and

$$
\begin{aligned}
\varrho\left(Y^{\lambda}\right) & =\mathrm{t}^{-(\rho, \lambda)} \varrho\left(T_{x^{-1}}^{-1}\right) x^{-1} t_{\lambda} y^{-1} \varrho\left(T_{y}\right) \\
& =\mathrm{t}^{-(\rho, \lambda)} \mathrm{G}_{\beta_{l}}^{-} \cdots \mathrm{G}_{\beta_{1}}^{-} t_{\lambda} \mathrm{G}_{\gamma_{1}}^{+} \cdots \mathrm{G}_{\gamma_{m}}^{+}
\end{aligned}
$$

in the polynomial representation. Thus we need to compute the limit

$$
\varrho_{0}^{\prime}\left(Y^{\lambda}\right)=\lim _{v \rightarrow 0} \varkappa\left(\mathrm{G}_{\beta_{l}}^{-} \cdots \mathrm{G}_{\beta_{1}}^{-}\right) \mathrm{t}^{-(\rho, \lambda)} \varkappa\left(t_{\lambda}\right) \varkappa\left(\mathrm{G}_{\gamma_{1}}^{+} \cdots \mathrm{G}_{\gamma_{m}}^{+}\right) .
$$

The matrix $\mathrm{t}^{-(\rho, \lambda)} \varkappa\left(t_{\lambda}\right)$ is diagonal with entries

$$
\mathrm{t}^{-(\rho, \lambda)} \varkappa\left(t_{\lambda}\right)_{w, w}=\mathrm{t}^{\left(\rho, w^{-1} \lambda\right)-(\rho, \lambda)} t_{w^{-1} \lambda} .
$$

Using equation (3.56), we can write $\mathrm{t}^{-(\rho, \lambda)} \varkappa\left(t_{\lambda}\right)=\varkappa_{\lambda}^{-} \tau_{\lambda} \varkappa_{\lambda}^{+}$, where $\varkappa_{\lambda}^{ \pm}$and $\tau_{\lambda}$ are the diagonal matrices given by

$$
\left(x_{\lambda}^{-}\right)_{w, w}=\mathrm{t}^{\ell_{\lambda}^{-}(w)}, \quad\left(\tau_{\lambda}\right)_{w, w}=t_{w^{-1} \lambda}, \quad\left(x_{\lambda}^{+}\right)_{w, w}=\mathrm{t}^{-\ell_{\lambda}^{+}(w)} .
$$

Our strategy is to commute $\varkappa_{\lambda}^{-}$to the left past $\varkappa\left(\mathrm{G}_{\beta_{l}}^{-} \cdots \mathrm{G}_{\beta_{1}}^{-}\right)$, and $\varkappa_{\lambda}^{+}$to the right past $\varkappa\left(\mathrm{G}_{\gamma_{1}}^{+} \cdots \mathrm{G}_{\gamma_{m}}^{+}\right)$. After we have done so, all negative powers of $v$ will disappear.

For $r=1, \ldots, l$, we have

$$
\varkappa\left(\mathrm{G}_{\beta_{r}}^{-}\right)_{w, w} \cdot \mathrm{t}_{\lambda, r}^{\ell_{\lambda, r}^{-}(w)} \approx \mathrm{t}^{\ell_{\lambda, r+1}^{-}(w)} \times \begin{cases}1-X^{-w^{-1} \beta_{r}} & \text { if }\left(\rho, w^{-1} \beta_{r}\right)=1, \\ 1 & \text { if }\left(\rho, w^{-1} \beta_{r}\right)>1, \\ 1 & \text { if } w^{-1} \beta_{r}<0,\end{cases}
$$

and

$$
\begin{aligned}
& \varkappa\left(\mathrm{G}_{\beta_{r}}^{-}\right)_{w, s_{\beta_{r}} w} \cdot \mathrm{t}_{\lambda, r}^{\ell_{\lambda}^{-}\left(s_{\beta_{r}} w\right)} \\
& \approx \mathrm{t}_{\lambda, r+1}^{\ell^{-}(w)} \times \begin{cases}\mathrm{t}^{-1+\ell_{\lambda, r}^{-}\left(s_{\beta r} w\right)-\ell_{\lambda, r}^{-}(w)} & \text { if } w^{-1} \beta_{r}>0, \\
-\mathrm{t}^{-\left(\rho, w^{-1} \beta_{r}\right)+\ell_{\lambda, r}^{-}\left(s_{\beta_{r}} w\right)-\ell_{\lambda, r}^{-}(w)} X^{w^{-1} \beta_{r}} & \text { if } w^{-1} \beta_{r}<0\end{cases} \\
& =\mathrm{t}_{\lambda, r+1}^{-}(w) \times \begin{cases}\mathrm{v}^{-1+\ell\left(s_{\beta_{r}} w\right)-\ell(w)} & \text { if } w^{-1} \beta_{r}>0, \\
-\mathrm{v}^{-\left(2 \rho, w^{-1} \beta_{r}\right)-1+\ell\left(s_{\beta_{r}} w\right)-\ell(w)} X^{w^{-1} \beta_{r}} & \text { if } w^{-1} \beta_{r}<0,\end{cases}
\end{aligned}
$$

where we use equations (3.59) to obtain the last equality. Notice that in all cases, each factor after the bracket involves no negative powers of $v$. Moreover, after commuting $x_{\lambda}^{-}$all the way to the left, we arrive at $\mathrm{t}_{\lambda, l+1}^{-}(w)=1$, for any $w \in W$. 
Similarly, for $s=1, \ldots, m$, we have

$$
\mathrm{t}^{-\ell_{\lambda, s}^{+}(w)} \cdot \varkappa\left(\mathrm{G}_{\gamma_{s}}^{+}\right)_{w, w} \approx \mathrm{t}^{-\ell_{\lambda, s+1}^{+}(w)} \times \begin{cases}1 & \text { if } w^{-1} \gamma_{s}>0, \\ 1-X^{w^{-1} \gamma_{s}} & \text { if }\left(\rho, w^{-1} \gamma_{s}\right)=-1, \\ 1 & \text { if }\left(\rho, w^{-1} \gamma_{s}\right)<-1,\end{cases}
$$

and

$$
\begin{aligned}
& \mathrm{t}^{-\ell_{\lambda, s}^{+}(w)} \cdot \varkappa\left(\mathrm{G}_{\gamma_{s}}^{+}\right)_{w, s_{\gamma_{s}} w} \\
& \approx \mathrm{t}^{-\ell_{\lambda, s+1}^{+}\left(s_{\gamma_{s}} w\right)} \times \begin{cases}-\mathrm{t}^{-1+\ell_{\lambda, s}^{+}\left(s_{\gamma_{s}} w\right)-\ell_{\lambda, s}^{+}(w)} & \text { if } w^{-1} \gamma_{s}>0, \\
\mathrm{t}^{-\left(\rho, w^{-1} \gamma_{s}\right)+\ell_{\lambda, s}^{+}\left(s_{\gamma_{s}} w\right)-\ell_{\lambda, s}^{+}(w)} X^{w^{-1} \gamma_{s}} & \text { if } w^{-1} \gamma_{s}<0\end{cases} \\
& =\mathrm{t}^{-\ell_{\lambda, s+1}^{+}\left(s_{\gamma_{s}} w\right)} \times \begin{cases}-\mathrm{v}^{-1+\ell\left(s_{\gamma_{s}} w\right)-\ell(w)} & \text { if } w^{-1} \gamma_{s}>0, \\
\mathrm{v}^{-\left(2 \rho, w^{-1} \gamma_{s}\right)-1+\ell\left(s_{\gamma_{s}} w\right)-\ell(w)} X^{w^{-1} \gamma_{s}} & \text { if } w^{-1} \gamma_{s}<0 .\end{cases}
\end{aligned}
$$

As before, each factor after the bracket involves no negative powers of $\mathrm{v}$, and after commuting $\varkappa_{\lambda}^{+}$all the way to the right, we arrive at $\mathrm{t}_{\lambda, m+1}^{+}(w)=1$, for any $w \in W$.

To complete the proof, we expand the product of resulting matrices (including $\tau_{\lambda}$ ) and take $v \rightarrow 0$. Taking into account the exponents of $\mathrm{v}$, one sees that the surviving terms are exactly those indexed by quantum walks $\mathbf{Q} \mathbf{W}_{\lambda, w}$.

\subsection{Inverse Chevalley formula}

By means of [25, Theorem 5.1], Theorem 3.7 immediately gives the following algebraic form of the inverse Chevalley formula in $K_{H \times \mathbb{C}^{*}}\left(\mathbf{Q}_{G}\right)$, which is our first main result:

Theorem 3.11. For any minuscule $\lambda \in P$ and $w \in W$, we have

$$
e^{\lambda} \cdot\left[\mathcal{O}_{\mathbf{Q}_{G}(w)}\right]=\sum_{\mathbf{w}=\left(w_{1}, \ldots, w_{n}\right) \in \mathbf{Q} \mathbf{W}_{\lambda, w}}\left[\mathcal{O}_{\mathbf{Q}_{G}\left(w_{n}\right)}\right] \cdot \widetilde{g}_{\mathbf{w}}^{+} X^{-w_{\circ} w_{l}^{-1} \lambda \widetilde{g}_{\mathbf{w}}^{-}}
$$

in terms of the q-Heisenberg action of Proposition 3.3, with

$$
\begin{aligned}
& \widetilde{g}_{\mathbf{w}}^{-}=\prod_{1 \leq t \leq l} \begin{cases}1-\widetilde{X}^{-w_{\circ} w_{t-1}^{-1} \eta_{t}} & \text { if } w_{t}=w_{t-1} \text { and }\left(\rho, w_{t-1}^{-1} \eta_{t}\right)=1, \\
-\widetilde{X}^{w_{\circ} w_{t-1}^{-1} \eta_{t}} & \text { if } w_{t}<w_{t-1}, \\
1 & \text { otherwise }\end{cases} \\
& \widetilde{g}_{\mathbf{w}}^{+}=\prod_{l<t \leq n} \begin{cases}1-\widetilde{X}^{w_{\circ} w_{t-1}^{-1} \eta_{t}} & \text { if } w_{t}=w_{t-1} \text { and }\left(\rho, w_{t-1}^{-1} \eta_{t}\right)=-1, \\
\widetilde{X}^{w_{\circ} w_{t-1}^{-1} \eta_{t}} & \text { if } w_{t}<w_{t-1}, \\
-1 & \text { if } w_{t}>w_{t-1}, \\
1 & \text { otherwise, }\end{cases}
\end{aligned}
$$

and $\widetilde{X}^{\beta}=q \cdot t_{\beta} X^{\beta}$ for $\beta \in \Delta$.

Remark 3.12. The elements $\widetilde{X}^{\beta}$ commute, as one easily checks.

Remark 3.13. The image of equation (3.62) under the map trunc of formula (1.3) recovers the corresponding inverse Chevalley formula in $K_{H}(G / B)$ given by [23, p. 239]; note that under the map trunc, the class $\left[\mathcal{O}_{\mathbf{Q}_{G}}(\mu)\right]$ for $\mu \in P$ corresponds to the class $\left[\mathcal{O}_{G / B}(\mu)\right]$, which is denoted by $[\mathcal{L}(-\mu)]$ in [23]. We note also that the image of equation (3.62) under trunc has positive coefficients for $\lambda \in-P_{+}$ 
(i.e., when $l=n$ ), and alternating coefficients for $\lambda \in P_{+}$(i.e., when $l=0$ ). Informally, the effect of the map trunc is achieved by setting $\widetilde{X}^{\beta}=0$ in equations (3.63) and (3.64), where $\beta \in \Delta^{+}$ always.

\subsubsection{Decorated quantum walks}

We now proceed to further combinatorialise equation (3.62). First we enlarge the summation set, so that each term on the right-hand side of equation (3.62) is a product of monomials in the $q$-Heisenberg algebra.

Given $\mathbf{w}=\left(w_{1}, \ldots, w_{n}\right) \in \mathbf{Q} \mathbf{W}_{\lambda, w}$, let $S^{-}(\mathbf{w})$ denote the set of steps $t$, for $1 \leq t \leq l$, such that $w_{t}=w_{t-1}$ and $\left(\rho, w_{t-1}^{-1} \eta_{t}\right)=1$. Similarly, let $S^{+}(\mathbf{w})$ denote the set of steps $t$, for $l<t \leq n$ such that $w_{t}=w_{t-1}$ and $\left(\rho, w_{t-1}^{-1} \eta_{t}\right)=-1$.

Let $S(\mathbf{w})=S^{-}(\mathbf{w}) \cup S^{+}(\mathbf{w})$, and define the set of decorated quantum walks $\widetilde{\mathbf{Q W}}_{\lambda, w}$ to consist of all pairs (w, b) where $\mathbf{w} \in \mathbf{Q} \mathbf{W}_{\lambda, w}$ and $\mathbf{b}$ is a $\{0,1\}$-valued function on $S(\mathbf{w})$. Then equation (3.62) can be written as

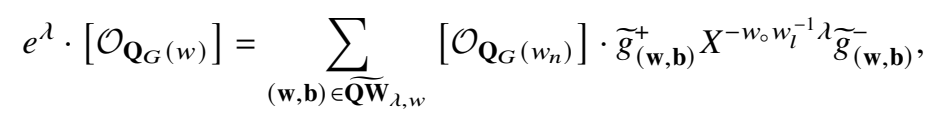

with

$$
\begin{gathered}
\widetilde{g}_{(\mathbf{w}, \mathbf{b})}^{-}=\prod_{1 \leq t \leq l} \begin{cases}\left(-\widetilde{X}^{-w_{\circ} w_{t-1}^{-1}} \eta_{t}\right. & \mathbf{b}(t) \\
-\widetilde{X}^{w_{\circ} w_{t-1}^{-1}} \eta_{t} & \text { if } t \in S^{-}(\mathbf{w}), \\
1 & \text { if } w_{t}<w_{t-1}, \\
\text { otherwise }\end{cases} \\
\widetilde{g}_{(\mathbf{w}, \mathbf{b})}^{+}=\prod_{l<t \leq n} \begin{cases}\left(-\widetilde{X}^{w_{\circ} w_{t-1}^{-1}} \eta_{t}\right) & \text { if } t \in S^{+}(\mathbf{w}), \\
\widetilde{X}^{w_{\circ} w_{t-1}^{-1} \eta_{t}} & \text { if } w_{t}<w_{t-1}, \\
-1 & \text { if } w_{t}>w_{t-1}, \\
1 & \text { otherwise. }\end{cases}
\end{gathered}
$$

Next, to expand equation (3.65) further, let us introduce some more notation. For $(\mathbf{w}, \mathbf{b}) \in \widetilde{\mathbf{Q W}}_{\lambda, w}$, define the sign

$$
(-1)^{(\mathbf{w}, \mathbf{b})}=\prod_{\substack{1 \leq t \leq l \\ w_{t}<w_{t-1}}}(-1) \prod_{\substack{l<t \leq n \\ w_{t}>w_{t-1}}}(-1) \prod_{t \in S(\mathbf{w})}(-1)^{\mathbf{b}(t)}
$$

and partial weights (in $Q$ )

$$
\begin{aligned}
& \mathrm{wt}_{0}(\mathbf{w}, \mathbf{b})=0 \\
& \mathrm{wt}_{t}(\mathbf{w}, \mathbf{b})=\mathrm{wt}_{t-1}(\mathbf{w}, \mathbf{b})+ \begin{cases}-\mathbf{b}(t) w_{\circ} w_{t-1}^{-1} \eta_{t} & \text { if } t \in S^{-}(\mathbf{w}), \\
w_{\circ} w_{t-1}^{-1} \eta_{t} & \text { if } w_{t}<w_{t-1}, \quad \text { for } 1 \leq t \leq l \\
0 & \text { otherwise }\end{cases} \\
& \mathrm{wt}_{t}(\mathbf{w}, \mathbf{b})=\mathrm{wt}_{t-1}(\mathbf{w}, \mathbf{b})+ \begin{cases}\mathbf{b}(t) w_{\circ} w_{t-1}^{-1} \eta_{t} & \text { if } t \in S^{+}(\mathbf{w}), \\
w_{\circ} w_{t-1}^{-1} \eta_{t} & \text { if } w_{t}<w_{t-1}, \quad \text { for } l<t \leq n . \\
0 & \text { otherwise }\end{cases}
\end{aligned}
$$


Define the weight of $(\mathbf{w}, \mathbf{b})$ to be $\operatorname{wt}(\mathbf{w}, \mathbf{b})=\mathrm{wt}_{n}(\mathbf{w}, \mathbf{b}) \in Q$, and set $d_{t}(\mathbf{w}, \mathbf{b})=\mathrm{wt}_{t}(\mathbf{w}, \mathbf{b})-$ $\mathrm{wt}_{t-1}(\mathbf{w}, \mathbf{b})$ for $1 \leq t \leq n$.

Define the partial degrees (in $\mathbb{Z}$ ):

$$
\begin{array}{ll}
\operatorname{deg}_{0}^{-}(\mathbf{w}, \mathbf{b})=0 & \\
\operatorname{deg}_{t}^{-}(\mathbf{w}, \mathbf{b})=\operatorname{deg}_{t-1}^{-}(\mathbf{w}, \mathbf{b})+\frac{\left|d_{t}(\mathbf{w}, \mathbf{b})\right|^{2}}{2}+\left(d_{t}(\mathbf{w}, \mathbf{b}), \mathrm{wt}_{t-1}(\mathbf{w}, \mathbf{b})\right), \quad \text { for } 1 \leq t \leq l \\
\operatorname{deg}_{l}^{+}(\mathbf{w}, \mathbf{b})=\operatorname{deg}_{l}^{-}(\mathbf{w}, \mathbf{b})+\left(-w_{\circ} w_{l}^{-1} \lambda, \mathrm{wt}_{l}(\mathbf{w}, \mathbf{b})\right) \\
\operatorname{deg}_{t}^{+}(\mathbf{w}, \mathbf{b})=\operatorname{deg}_{t-1}^{+}(\mathbf{w}, \mathbf{b})+\frac{\left|d_{t}(\mathbf{w}, \mathbf{b})\right|^{2}}{2}+\left(d_{t}(\mathbf{w}, \mathbf{b}), \mathrm{wt}_{t-1}(\mathbf{w}, \mathbf{b})\right), \quad \text { for } l<t \leq n .
\end{array}
$$

Define $\operatorname{deg}(\mathbf{w}, \mathbf{b})=\operatorname{deg}_{n}^{+}(\mathbf{w}, \mathbf{b}) \in \mathbb{Z}$.

Then, from equation (3.65), we obtain our second main result, the combinatorial form of our inverse Chevalley formula:

Theorem 3.14. For any minuscule $\lambda \in P$ and $w \in W$, we have

$$
\begin{aligned}
& e^{\lambda} \cdot\left[\mathcal{O}_{\mathbf{Q}_{G}(w)}\right] \\
& =\sum_{(\mathbf{w}, \mathbf{b}) \in \widetilde{\mathbf{Q W}}_{\lambda, w}}(-1)^{(\mathbf{w}, \mathbf{b})} q^{\operatorname{deg}(\mathbf{w}, \mathbf{b})} \cdot\left[\mathcal{O}_{\mathbf{Q}_{G}\left(w_{n} t_{-w_{\circ}(\mathrm{wt}(\mathbf{w}, \mathbf{b}))}\right)}\left(-w_{\circ} w_{l}^{-1} \lambda+\mathrm{wt}(\mathbf{w}, \mathbf{b})\right)\right] .
\end{aligned}
$$

Proof. Proceed from right to left in equation (3.65), commuting all translations to the left of line bundle twists, and then act on $\left[\mathcal{O}_{\mathbf{Q}_{G}\left(w_{n}\right)}\right]$.

\section{Appendix A. The type $A$ case}

We briefly consider the type $A$ case. For this appendix, let $G=S L(n+1, \mathbb{C})$.

\section{A.1. The root system of type $A$}

Let $\left\{\varepsilon_{i}: 1 \leq i \leq n+1\right\}$ be the standard basis of $\mathbb{Z}^{n+1}$. We realise the weight lattice as $P=\mathbb{Z}^{n+1} / \mathbb{Z}\left(\varepsilon_{1}+\right.$ $\left.\cdots+\varepsilon_{n+1}\right)$, and by abuse of notation, we continue to denote the image of $\varepsilon_{i}$ in $P$ by the same symbol. Thus $\varpi_{k}:=\varepsilon_{1}+\cdots+\varepsilon_{k}$, for $k \in\{1, \ldots, n\}$, are the fundamental weights of $G$.

We set $\alpha_{i}:=\varepsilon_{i}-\varepsilon_{i+1}$ for $i \in\{1, \ldots, n\}$ and $\alpha_{i, j}:=\alpha_{i}+\alpha_{i+1}+\cdots+\alpha_{j}$ for $i, j \in\{1, \ldots, n\}$ with $i \leq j$. Then the root system is $\Delta:=\left\{ \pm \alpha_{i, j} \mid 1 \leq i \leq j \leq n\right\}$, with the set of positive roots $\Delta^{+}:=\left\{\alpha_{i, j} \mid 1 \leq i \leq j \leq n\right\}$ and the set of simple roots $\left\{\alpha_{1}, \ldots, \alpha_{n}\right\}$.

We identify the Weyl group $W$ with the symmetric group $\mathfrak{\subseteq}_{n+1}$ in the usual way. Regarding $w \in W$ as a permutation, we have $w \varepsilon_{i}=\varepsilon_{w(i)}$ for $i \in\{1, \ldots, n+1\}$. Thus, for $i, j \in\{1, \ldots, n\}$ with $i \leq j$, the reflection $s_{\alpha_{i, j}}$ corresponds to the transposition $(i, j+1) \in \mathfrak{S}_{n+1}$. The longest element of $W$ is given by $w_{\circ}(i)=n+2-i$ for $i \in\{1, \ldots, n+1\}$.

\section{A.2. Generators}

Each fundamental weight $\varpi_{k}(k=1, \ldots, n)$ is minuscule. Moreover, we have $w_{\circ} \varpi_{k}=-\varpi_{n+1-k}$. Since the $\varepsilon_{i}$ generate $P$, the inverse Chevalley rule in $K_{H \times \mathbb{C}^{*}}\left(\mathbf{Q}_{G}^{\text {rat }}\right)$ is completely determined by Theorem 3.14 in the case of $\lambda= \pm \varepsilon_{i}$, where $1 \leq i \leq n+1$. (That is, $\lambda$ belongs to the $W$-orbit of either $\varpi_{1}$ or $\varpi_{n}=-\varepsilon_{n+1}$.)

\section{A.3. Examples}

We give two concrete examples of our inverse Chevalley formula (3.62) in type $A$. 
18

Takafumi Kovno et al.

First, for $G=S L(3, \mathbb{C})$ and $\lambda=\varpi_{1}$, we have

$$
\begin{aligned}
e^{\varpi_{1}} \cdot\left[\mathcal{O}_{\mathbf{Q}_{G}\left(s_{1}\right)}\right]=[ & \left.\mathcal{O}_{\mathbf{Q}_{G}\left(s_{1}\right)}\left(-w_{\circ} s_{1} \varpi_{1}\right)\right]-q\left[\mathcal{O}_{\mathbf{Q}_{G}\left(s_{1} t_{\alpha_{1}}\right)}\left(-w_{\circ} \varpi_{1}\right)\right] \\
& -\left[\mathcal{O}_{\mathbf{Q}_{G}\left(s_{1} s_{2}\right)}\left(-w_{\circ} s_{1} \varpi_{1}\right)\right]+q\left[\mathcal{O}_{\mathbf{Q}_{G}\left(t_{\alpha_{1}}\right)}\left(-w_{\circ} \varpi_{1}\right)\right] .
\end{aligned}
$$

Indeed, $\left\lfloor w_{\circ}\right\rfloor=s_{2} s_{1}, l=0, \vec{\eta}=\left(\gamma_{1}, \gamma_{2}\right)=\left(\alpha_{1}+\alpha_{2}, \alpha_{1}\right)$ and three of the four possible walks are quantum walks, namely

$$
\mathbf{Q} \mathbf{W}_{\varpi_{1}, s_{1}}=\left\{\left(s_{1}, s_{1}\right),\left(s_{1} s_{2}, s_{1} s_{2}\right),\left(s_{1}, e\right)\right\} .
$$

(The reason the remaining walk $\left(s_{1} s_{2}, s_{2}\right) \in \mathbf{W}_{\varpi_{1}, s_{1}}$ is not a quantum walk is that $s_{1} s_{2} \rightarrow s_{2}$ is not an edge in $\mathrm{QBG}(W)$.) The right-hand side of equation (3.62) produces the following expression for $e^{\varpi_{1}} \cdot\left[\mathcal{O}_{\mathbf{Q}_{G}\left(s_{1}\right)}\right]:$

$$
\left(\left[\mathcal{O}_{\mathbf{Q}_{G}\left(s_{1}\right)}\right] \cdot \widetilde{g}_{\left(s_{1}, s_{1}\right)}^{+}+\left[\mathcal{O}_{\mathbf{Q}_{G}\left(s_{1} s_{2}\right)}\right] \cdot \widetilde{g}_{\left(s_{1} s_{2}, s_{1} s_{2}\right)}^{+}+\left[\mathcal{O}_{\mathbf{Q}_{G}(e)}\right] \cdot \widetilde{g}_{\left(s_{1}, e\right)}^{+}\right) \cdot X^{-w_{o} s_{1} \varpi_{1}},
$$

where the $q$-Heisenberg elements are

$$
\begin{aligned}
\widetilde{g}_{\left(s_{1}, s_{1}\right)}^{+} & =1-\widetilde{X}^{w_{\circ} s_{1} \alpha_{1}}=1-q t_{-w_{\circ} \alpha_{1}} X^{-w_{\circ} \alpha_{1}}, \\
\widetilde{g}_{\left(s_{1} s_{2}, s_{1} s_{2}\right)}^{+} & =-1, \\
\widetilde{g}_{\left(s_{1}, e\right)}^{+} & =\widetilde{X}^{w_{\circ} s_{1} \alpha_{1}}=q t_{-w_{\circ} \alpha_{1}} X^{-w_{\circ} \alpha_{1}} .
\end{aligned}
$$

Finally, we use the formulas in Proposition 3.3 to obtain equation (A.1).

For our second example, we consider $G=S L(4, \mathbb{C})$ and $\lambda=s_{2} \varpi_{2}=\varepsilon_{1}+\varepsilon_{3}$. One has $\left\lfloor w_{\circ}\right\rfloor=s_{2} s_{1} s_{3} s_{2}$, $l=1$ and $\vec{\eta}=\left(\beta_{1}, \gamma_{1}, \gamma_{2}, \gamma_{3}\right)=\left(\alpha_{2}, \alpha_{1}+\alpha_{2}+\alpha_{3}, \alpha_{1}, \alpha_{3}\right)$. Using one-line notation for permutations in $W=S_{4}$, equation (3.62) gives

$$
\begin{aligned}
e^{\varepsilon_{1}+\varepsilon_{3}} \cdot\left[\mathcal{O}_{\mathbf{Q}_{G}(4312)}\right]= & {\left[\mathcal{O}_{\mathbf{Q}_{G}(4312)}\right] \cdot\left(1-\widetilde{X}^{-w_{\circ} \alpha_{1}}\right) X^{-w_{\circ} w^{-1} \lambda} } \\
& +\left[\mathcal{O}_{\mathbf{Q}_{G}(3412)}\right] \cdot \widetilde{X}^{-w_{\circ} \alpha_{1}} X^{-w_{\circ} w^{-1} \lambda} \\
& -\left[\mathcal{O}_{\mathbf{Q}_{G}(4321)}\right] \cdot\left(1-\widetilde{X}^{-w_{\circ} \alpha_{1}}\right) X^{-w_{\circ} w^{-1} \lambda} \\
& -\left[\mathcal{O}_{\mathbf{Q}_{G}(3421)}\right] \cdot \widetilde{X}^{-w_{\circ} \alpha_{1}} X^{-w_{\circ} w^{-1} \lambda} \\
& +\left[\mathcal{O}_{\mathbf{Q}_{G}(1342)}\right] \cdot \widetilde{X}^{-w_{\circ}\left(\alpha_{1}+\alpha_{2}\right)} X^{-w_{\circ} w^{-1} \lambda} \\
& -\left[\mathcal{O}_{\mathbf{Q}_{G}(1432)}\right] \cdot \widetilde{X}^{-w_{\circ}\left(\alpha_{1}+\alpha_{2}\right)} X^{-w_{\circ} w^{-1} \lambda} \\
& -\left[\mathcal{O}_{\mathbf{Q}_{G}(2341)}\right] \cdot \widetilde{X}^{-w_{\circ}\left(\alpha_{1}+\alpha_{2}\right)} X^{-w_{\circ} w^{-1} \lambda} \\
& +\left[\mathcal{O}_{\mathbf{Q}_{G}(2431)}\right] \cdot \widetilde{X}^{-w_{\circ}\left(\alpha_{1}+\alpha_{2}\right)} X^{-w_{\circ} w^{-1} \lambda}
\end{aligned}
$$

where $w=4312$. We note that $\widetilde{g}_{\mathbf{w}}^{-}=1$ and $w_{l}=w$ for all quantum walks in $\mathbf{Q W}{\boldsymbol{\varepsilon}_{1}+\varepsilon_{2}, 4312}_{\text {. Using }}$ Proposition 3.3, we obtain

$$
\begin{aligned}
e^{\varepsilon_{1}+\varepsilon_{3}} \cdot\left[\mathcal{O}_{\mathbf{Q}_{G}(4312)}\right]= & {\left[\mathcal{O}_{\mathbf{Q}_{G}(4312)}\left(-w_{\circ} w^{-1} \lambda\right)\right]-q\left[\mathcal{O}_{\mathbf{Q}_{G}\left(4312 \cdot t_{\alpha_{1}}\right.}\left(-w_{\circ}\left(w^{-1} \lambda+\alpha_{1}\right)\right)\right] } \\
& +q\left[\mathcal{O}_{\mathbf{Q}_{G}\left(3412 \cdot t_{\alpha_{1}}\right)}\left(-w_{\circ}\left(w^{-1} \lambda+\alpha_{1}\right)\right)\right] \\
& -\left[\mathcal{O}_{\mathbf{Q}_{G}(4321)}\left(-w_{\circ} w^{-1} \lambda\right)\right]+q\left[\mathcal{O}_{\mathbf{Q}_{G}\left(4321 \cdot t_{\alpha_{1}}\right)}\left(-w_{\circ}\left(w^{-1} \lambda+\alpha_{1}\right)\right)\right] \\
& -q\left[\mathcal{O}_{\mathbf{Q}_{G}\left(3421 \cdot t_{\alpha_{1}}\right)}\left(-w_{\circ}\left(w^{-1} \lambda+\alpha_{1}\right)\right)\right] \\
& +q\left[\mathcal{O}_{\mathbf{Q}_{G}\left(1342 \cdot t_{\alpha_{1}+\alpha_{2}}\right)}\left(-w_{\circ}\left(w^{-1} \lambda+\alpha_{1}+\alpha_{2}\right)\right)\right]
\end{aligned}
$$

https://doi.org/10.1017/fms.2021.45 Published online by Cambridge University Press 


$$
\begin{aligned}
& -q\left[\mathcal{O}_{\mathbf{Q}_{G}\left(1432 \cdot t_{\alpha_{1}+\alpha_{2}}\right)}\left(-w_{\circ}\left(w^{-1} \lambda+\alpha_{1}+\alpha_{2}\right)\right)\right] \\
& -q\left[\mathcal{O}_{\mathbf{Q}_{G}\left(2341 \cdot t_{\alpha_{1}+\alpha_{2}}\right)}\left(-w_{\circ}\left(w^{-1} \lambda+\alpha_{1}+\alpha_{2}\right)\right)\right] \\
& +q\left[\mathcal{O}_{\mathbf{Q}_{G}\left(2431 \cdot t_{\alpha_{1}+\alpha_{2}}\right)}\left(-w_{\circ}\left(w^{-1} \lambda+\alpha_{1}+\alpha_{2}\right)\right)\right],
\end{aligned}
$$

where $w^{-1} \lambda=\varepsilon_{2}+\varepsilon_{3}, w^{-1} \lambda+\alpha_{1}=\varepsilon_{1}+\varepsilon_{3}$ and $w^{-1} \lambda+\alpha_{1}+\alpha_{2}=\varepsilon_{1}+\varepsilon_{2}$.

\section{A.4. Special case: $w=w_{\circ}$ and $k=1$}

For $G=S L(n+1, \mathbb{C})$, we consider the special case $w=w_{\circ}$ and $k=1$; the latter means that $\lambda=\varepsilon_{l+1}$ for some $0 \leq l \leq n$. In the setting of Section 3.3.3, we have

$$
\begin{aligned}
\left\lfloor w_{\circ}\right\rfloor & =s_{n} \cdots s_{1} \\
& =\underbrace{s_{n} \cdots s_{l+1}}_{=y} \underbrace{s_{l} \cdots s_{1}}_{=x}
\end{aligned}
$$

and $\vec{\eta}=\left(\eta_{1}, \ldots, \eta_{n}\right)=\left(\beta_{l}, \ldots, \beta_{1}, \gamma_{1}, \ldots, \gamma_{m}\right)$, where $m=\ell(y)=n-l$ and

$$
\begin{aligned}
& \beta_{r}=\varepsilon_{r}-\varepsilon_{l+1}, \quad 1 \leq r \leq l, \\
& \gamma_{s}=\varepsilon_{l+1}-\varepsilon_{n+2-s}, \quad 1 \leq s \leq m .
\end{aligned}
$$

Let us abbreviate our notation as follows: $\mathbf{W}_{w}^{(l)}=\mathbf{W}_{\varepsilon_{l+1}, w}$ and $\mathbf{Q} \mathbf{W}_{w}^{(l)}=\mathbf{Q} \mathbf{W}_{\varepsilon_{l+1}, w}$.

The following lemma describes the set $\mathbf{Q} \mathbf{W}_{w_{0}}^{(l)}$. Its proof is straightforward and is left to the reader.

Lemma A.1. A walk $\mathbf{w} \in \mathbf{W}_{w_{\circ}}^{(l)}$ belongs to $\mathbf{Q} \mathbf{W}_{w_{\circ}}^{(l)}$ if and only if one of the following holds:

(a) $\mathbf{w}=\left(w_{1}, \ldots, w_{l}, w_{l}, \ldots, w_{l}\right)$, where $\left(w_{1}, \ldots, w_{l}\right) \in \mathbf{W}_{w_{\circ}}^{\left(\alpha_{l, l}, \ldots, \alpha_{1, l}\right)}$.

(b) $\mathbf{w}=\left(w_{\circ}, \ldots, w_{\circ}, w_{l+1}, \ldots, w_{n}\right)$, where $\left(w_{l+1}, \ldots, w_{n}\right) \in \mathbf{W}_{w_{\circ}}^{\left(\alpha_{l+1, n}, \ldots, \alpha_{l+1, l+1}\right)}$.

We have $S^{-}(\mathbf{w})=\varnothing$ in all cases, and $S^{+}(\mathbf{w})=\varnothing$ unless $l<n$ and $\mathbf{w}=\left(w_{\circ}, \ldots, w_{\circ}\right)$, in which case $S^{+}(\mathbf{w})=\{n\}$. (Note that the two cases in the lemma share the walk $\mathbf{w}=\left(w_{\circ}, \ldots, w_{\circ}\right)$.)

Combining Lemma A.1 with Theorem 3.11, one obtains the following (where we set $i=l+1$ ):

Proposition A.2. In $K_{H \times \mathbb{C}^{*}}\left(\mathbf{Q}_{G}\right)$ for $G=S L(n+1, \mathbb{C})$, one has

$$
\begin{aligned}
& e^{\varepsilon_{i}} \cdot\left[\mathcal{O}_{\left.\mathbf{Q}_{G}\left(w_{\circ}\right)\right]=}\right. {\left[\mathcal{O}_{\mathbf{Q}_{G}\left(w_{\circ}\right)}\left(-\varepsilon_{i}\right)\right]-\mathbf{1}_{\{i<n+1\}} \cdot q \cdot\left[\mathcal{O}_{\mathbf{Q}_{G}\left(w_{\circ} t_{-w_{0}\left(\alpha_{i}\right)}\right)}\left(-\varepsilon_{i+1}\right)\right] } \\
&+\sum_{\varnothing \neq\left\{i_{1}<\cdots<i_{a}\right\} \subset\{1, \ldots, i-1\}}(-1)^{a}\left[\mathcal{O} \mathcal{Q}_{G}\left(\left(i_{1} \cdots i_{a}\right)^{-1} w_{\circ} t_{-w_{\circ}\left(\alpha_{i_{1}, i-1}\right)}\right)\right. \\
&+\sum_{\varnothing \neq\left\{j_{1}<\cdots<j_{b}\right\} \subset\{i+1, n+1\}}(-1)^{b-1} q \cdot\left[\mathcal{O}_{\left.\mathbf{Q}_{G}\right)}\left(\left(i j_{1} \cdots j_{b}\right)^{-1} w_{\circ} t_{-w_{\circ}\left(\alpha_{i, j_{b}-1}\right)}\right)\right. \\
&
\end{aligned}
$$

where $1 \leq i \leq n+1$ and

$$
\mathbf{1}_{\{i<n+1\}}= \begin{cases}1 & \text { if } i<n+1 \\ 0 & \text { otherwise. }\end{cases}
$$


Remark A.3. The leading term $\left[\mathcal{O}_{\mathbf{Q}_{G}\left(w_{\circ}\right)}\left(-\varepsilon_{i}\right)\right]$ in equation (A.7) recovers the 'classical' analogue of this formula in $K_{H}(G / B)$, via the map trunc of formula (1.3), while the remaining terms give the explicit corrections in $K_{H \times \mathbb{C}^{*}}\left(\mathbf{Q}_{G}\right)$.

Remark A.4. Applying the diagram automorphism ' $-w_{\circ}$,' which sends

$$
\left[\mathcal{O}_{\mathbf{Q}_{G}\left(w t_{\beta}\right)}(\mu)\right] \mapsto\left[\mathcal{O}_{\mathbf{Q}_{G}\left(w_{\circ} w w_{\circ} t_{-w_{\circ}(\beta)}\right)}\left(-w_{\circ} \mu\right)\right],
$$

and an equivariant scalar $e^{\lambda} \mapsto e^{-w_{0} \lambda}$, one obtains a similar formula for $e^{-\varepsilon_{n+2-i}} \cdot\left[\mathcal{O}_{\mathbf{Q}_{G}\left(w_{\circ}\right)}\right]$ in $K_{H \times \mathbb{C}^{*}}\left(\mathbf{Q}_{G}\right)$. By the observations in Section 3.1.4, the action of $e^{\lambda}$ on $K_{H \times \mathbb{C}^{*}}\left(\mathbf{Q}_{G}\right)$ for any $\lambda \in P$ is then completely determined by iteration of these two formulas together with the $D_{i}$ for $i \in I$.

\section{A.5. Symmetrisation}

The usual $q$-Toda difference operators are realised geometrically by the 'spherical part' of $\mathbb{K}$, which is the free $\mathfrak{H}$-submodule generated by $\left[\mathcal{O}_{\mathbf{Q}_{G}}\right]$. By [25, Corollary 5.3], the action (of a spherical nil-affine Hecke subalgebra of $\left.\mathbb{H}_{0}\right)$ on the spherical part is obtained by taking the $(e, e)$-entry of the corresponding matrix in $\operatorname{Mat}_{W}(\mathfrak{S})$, namely

$$
f(X) \cdot\left[\mathcal{O}_{\mathbf{Q}_{G}}\right]=\left[\mathcal{O}_{\mathbf{Q}_{G}}\right] \cdot \varrho_{0}(f)_{e, e},
$$

for any symmetric Laurent polynomial $f=f(X) \in \mathbb{Z}\left[q^{ \pm 1}\right][X]^{W} \subset \mathbb{H}_{0}$.

Applying equation (A.9) for $G=S L(n+1, \mathbb{C})$ to the symmetrisation of $e^{\varpi_{1}}$, one obtains

$$
\sum_{i=1}^{n+1} e^{\varepsilon_{i}} \cdot\left[\mathcal{O}_{\mathbf{Q}_{G}}\right]=\left[\mathcal{O}_{\mathbf{Q}_{G}}\right] \cdot \varrho_{0}\left(\sum_{i=1}^{n+1} X^{-\varepsilon_{i}}\right)_{e, e}
$$

and we can use equation (3.62) to compute the right-hand side. One can easily show that just a single term contributes to the $(e, e)$-entry for each $i=1, \ldots, n+1$. The contributing terms are those given by the stationary walk $(e, \ldots, e) \in \mathbf{Q} \mathbf{W}_{e}^{(i-1)}$. Thus equation (3.62) immediately results in the following:

$$
\sum_{i=1}^{n+1} e^{\varepsilon_{i}} \cdot\left[\mathcal{O}_{\mathbf{Q}_{G}}\right]=\left[\mathcal{O}_{\mathbf{Q}_{G}}\right] \cdot\left(X^{-w_{\circ} \varepsilon_{1}}+\sum_{i=2}^{n+1} X^{-w_{\circ} \varepsilon_{i}}\left(1-q \cdot t_{-w_{\circ} \alpha_{i-1}} X^{-w_{\circ} \alpha_{i-1}}\right)\right) .
$$

The element of $\mathfrak{S}$ acting on the right-hand side of equation (A.11) is an equivalent form of the usual first-order $q$-Toda difference operator (compare [5, equation (2)]).

\section{Appendix B. Proofs of Lemma 3.8 and Proposition 3.9}

Recall the setting of Section 3.3.3. Equation (3.55) follows from the formula

$$
\rho-w^{-1} \rho=\sum_{\alpha \in \operatorname{Inv}\left(w^{-1}\right)} \alpha \quad \text { for } w \in W
$$

applied to the cases $w=x$ and $w=\left\lfloor w_{\circ}\right\rfloor$, by using the fact that $\left(\alpha, \varpi_{k}\right)=1$ for all $\alpha \in \operatorname{Inv}\left(w^{-1}\right) \subset$ $\Delta^{+} \backslash \Delta_{J}^{+}$with $w \in W^{J}$. Equation (3.56) follows from equation (B.1), using the fact that $(\lambda, \gamma) \in\{0, \pm 1\}$ for all $\gamma \in \Delta$.

The following lemma is well known; indeed, it follows from the Cauchy - Schwarz inequality:

Lemma B.1. For $\alpha, \beta \in \Delta$, we have $(\alpha, \beta) \in\{0, \pm 1, \pm 2\}$. In addition, $(\alpha, \beta)= \pm 2$ if and only if $\alpha= \pm \beta$.

Lemma B.2. 1. $\left(\lambda, \beta_{r}\right)=-1$ for all $1 \leq r \leq l$.

2. $\left(\beta_{r}, \beta_{t}\right) \in\{0,1\}$ for all $1 \leq r, t \leq l$ with $r \neq t$. 
Proof. (1) We see that

$$
\begin{aligned}
& \left(\lambda, \beta_{r}\right)=\left(s_{j_{l}} s_{j_{l-1}} \cdots s_{j_{2}} s_{j_{1}} \varpi_{k}, s_{j_{l}} s_{j_{l-1}} \cdots s_{j_{r+1}}\left(\alpha_{j_{r}}\right)\right) \\
& =(\underbrace{s_{j_{r}} s_{j_{r-1}} \cdots s_{j_{2}} s_{j_{1}}}_{=: v} \varpi_{k}, \alpha_{j_{r}})=\left(\varpi_{k}, v^{-1} \alpha_{j_{r}}\right) \text {. }
\end{aligned}
$$

Since $v=s_{j_{r}} s_{j_{r-1}} \cdots s_{j_{2}} s_{j_{1}} \in W^{J}$ with $s_{j_{r}} v<v$, it follows that $v^{-1} \alpha_{j_{r}} \in-\left(\Delta^{+} \backslash \Delta_{J}^{+}\right)$. Therefore, we have $\left(\varpi_{k}, v^{-1} \alpha_{j_{r}}\right)<0$; since $\varpi_{k}$ is minuscule, we obtain $\left(\varpi_{k}, v^{-1} \alpha_{j_{r}}\right)=-1$, as desired.

(2) By Lemma B.1, we have $\left(\beta_{r}, \beta_{t}\right) \in\{0, \pm 1\}$. Suppose, for a contradiction, that $\left(\beta_{r}, \beta_{t}\right)=-1$. Then $s_{\beta_{r}}\left(\beta_{t}\right)=\beta_{r}+\beta_{t} \in \Delta$. By (1), we have $\left(\lambda, \beta_{r}+\beta_{t}\right)=-2$, which contradicts the assumption that $\varpi_{k}$ is minuscule. This proves the lemma.

Equation (3.57) follows from Lemma B.2(1). Equation (3.58) follows from equation (3.57) using the Dynkin diagram automorphism $\omega: I \rightarrow I$ induced by $w_{\circ}$ (see Appendix B.2).

In Appendices B.1 and B.2, we provide a complete proof of Proposition 3.9, since we do not know of a suitable reference for these assertions.

\section{B.1. Proof of equation (3.59)}

For $\alpha=\sum_{i \in I} c_{i} \alpha_{i} \in Q$, we set ht $(\alpha):=\sum_{i \in I} c_{i} \in \mathbb{Z}$.

Lemma B.3. Set $1 \leq r<t \leq l$. If $\left(\beta_{r}, \beta_{t}\right)=1$, then $\operatorname{ht}\left(\beta_{r}\right)>\operatorname{ht}\left(\beta_{t}\right)$.

Proof. We prove the assertion by induction on $l=\ell(x)$. If $l=0$ or $l=1$, then the assertion is obvious. Assume that $l>1$. If $t=l$, then $\beta_{t}=\beta_{l}=\alpha_{j_{l}}$, and

$$
s_{j_{l-1}} \cdots s_{j_{r+1}}\left(\alpha_{j_{r}}\right)=s_{j_{l}} \beta_{r}=s_{\beta_{t}} \beta_{r}=\beta_{r}-\beta_{t} .
$$

Hence $\beta_{r}=\beta_{t}+s_{j_{l-1}} \cdots s_{j_{r+1}}\left(\alpha_{j_{r}}\right)$; note that $s_{j_{l-1}} \cdots s_{j_{r+1}}\left(\alpha_{j_{r}}\right) \in \Delta^{+}$, since $s_{j_{l-1}} \cdots s_{j_{r+1}} s_{j_{r}}$ is reduced. Therefore, we obtain $\mathrm{ht}\left(\beta_{r}\right)>\operatorname{ht}\left(\beta_{t}\right)$.

Assume that $t \leq l-1$. Note that $x^{\prime}:=s_{j_{l}} x \in W^{J}$ with $\ell\left(x^{\prime}\right)=\ell(x)-1$, and that $x^{\prime}=s_{j_{p-1}} \cdots s_{j_{2}} s_{j_{1}}$ is a reduced expression for $x^{\prime}$. We set

$$
\beta_{u}^{\prime}:=s_{j_{l-1}} \cdots s_{j_{u+1}}\left(\alpha_{j_{u}}\right)=s_{j_{l}} \beta_{u} \quad \text { for } 1 \leq u \leq l-1 .
$$

Since $1 \leq r<t \leq l-1$ satisfy the condition that $\left(\beta_{r}^{\prime}, \beta_{t}^{\prime}\right)=\left(\beta_{r}, \beta_{t}\right)=1$, it follows by our induction hypothesis that ht $\left(\beta_{r}^{\prime}\right)>$ ht $\left(\beta_{t}^{\prime}\right)$. Here we remark that

$$
\beta_{r}=\beta_{r}^{\prime}-\underbrace{\left(\beta_{r}^{\prime}, \alpha_{j_{l}}\right)}_{=: a} \alpha_{j_{l}}, \quad \beta_{t}=\beta_{t}^{\prime}-\underbrace{\left(\beta_{t}^{\prime}, \alpha_{j_{l}}\right)}_{=: b} \alpha_{j_{l}},
$$

where $a, b \in\{0,-1\}$ by Lemma B.2(2). If $a=-1$ or if $a=b=0$, then it is obvious that ht $\left(\beta_{r}\right)>\operatorname{ht}\left(\beta_{t}\right)$, since ht $\left(\beta_{r}^{\prime}\right)>$ ht $\left(\beta_{t}^{\prime}\right)$ by our induction hypothesis. Assume that $a=0$ and $b=-1$. Suppose, for a contradiction, that ht $\left(\beta_{r}\right) \leq \operatorname{ht}\left(\beta_{t}\right)$. Since ht $\left(\beta_{t}\right)=\mathrm{ht}\left(\beta_{t}^{\prime}\right)+1$ and $\operatorname{ht}\left(\beta_{r}\right)=\mathrm{ht}\left(\beta_{r}^{\prime}\right)$, and since ht $\left(\beta_{r}^{\prime}\right)>$ ht $\left(\beta_{t}^{\prime}\right)$ by our induction hypothesis, we have $\operatorname{ht}\left(\beta_{r}\right)=\operatorname{ht}\left(\beta_{t}\right)$. Since $\left(\beta_{r}, \beta_{t}\right)=1$ by the assumption, we see that $\beta_{r}-\beta_{t} \in \Delta$. However, $\operatorname{ht}\left(\beta_{r}-\beta_{t}\right)=\operatorname{ht}\left(\beta_{r}\right)-\operatorname{ht}\left(\beta_{t}\right)=0$, which is a contradiction. Hence we conclude that $\operatorname{ht}\left(\beta_{r}\right)>\operatorname{ht}\left(\beta_{t}\right)$, as desired. This proves the lemma.

Lemma B.4. Set $1 \leq r \leq l$, and set $h:=\operatorname{ht}\left(\beta_{r}\right)$. Then

$$
\begin{gathered}
\#\left\{r<t \leq l \mid\left(\beta_{t}, \beta_{r}\right)=1\right\}=h-1, \\
\#\left\{\alpha \in \Delta^{+} \mid \operatorname{ht}(\alpha)<\operatorname{ht}\left(\beta_{r}\right),\left(\alpha, \beta_{r}\right)=1\right\}=2(h-1) .
\end{gathered}
$$


Proof. We prove the assertion by induction on $l=\ell(x)$. If $l=0$ or $l=1$, then the assertion is obvious. Assume that $l>1$. If $r=l$, then the assertion is obvious. Assume now that $1 \leq r \leq l-1$. Note that $x^{\prime}:=s_{j_{l}} x \in W^{J}$ with $\ell\left(x^{\prime}\right)=\ell(x)-1$, and that $x^{\prime}=s_{j_{l-1}} \cdots s_{j_{2}} s_{j_{1}}$ is a reduced expression for $x^{\prime}$. We set

$$
\beta_{u}^{\prime}:=s_{j_{l-1}} \cdots s_{j_{u+1}}\left(\alpha_{j_{u}}\right)=s_{j_{l}} \beta_{u} \quad \text { for } 1 \leq u \leq l-1 .
$$

Since $\beta_{l}=\alpha_{j_{l}}$, we see by Lemma B.2(2) that $\left(\beta_{r}^{\prime}, \alpha_{j_{l}}\right)=-\left(\beta_{r}, \alpha_{j_{l}}\right)=-\left(\beta_{r}, \beta_{l}\right) \in\{0,-1\}$.

Case 1. Assume first that $\left(\beta_{r}^{\prime}, \alpha_{j_{l}}\right)=0$, or equivalently, $\left(\beta_{r}, \alpha_{j_{l}}\right)=0$; in this case, $\beta_{r}=\beta_{r}^{\prime}$, and hence ht $\left(\beta_{r}^{\prime}\right)=\operatorname{ht}\left(\beta_{r}\right)=h$. In addition, since $\beta_{l}=\alpha_{j_{l}}$, it follows that

$$
\begin{aligned}
\left\{r<t \leq l \mid\left(\beta_{t}, \beta_{r}\right)=1\right\} & =\left\{r<t \leq l-1 \mid\left(\beta_{t}, \beta_{r}\right)=1\right\} \\
& =\left\{r<t \leq l-1 \mid\left(s_{j_{l}} \beta_{t}, s_{j_{l}} \beta_{r}\right)=1\right\} \\
& =\left\{r<t \leq l-1 \mid\left(\beta_{t}^{\prime}, \beta_{r}^{\prime}\right)=1\right\} .
\end{aligned}
$$

By our induction hypothesis, we have $\#\left\{r<t \leq l-1 \mid\left(\beta_{t}^{\prime}, \beta_{r}^{\prime}\right)=1\right\}=h-1$, and hence $\#\{r<t \leq l \mid$ $\left.\left(\beta_{t}, \beta_{r}\right)=1\right\}=h-1$, as desired. Also, since $\beta_{r}=\beta_{r}^{\prime}$ in this case, it is obvious that

$$
\left\{\alpha \in \Delta^{+} \mid \operatorname{ht}(\alpha)<\operatorname{ht}\left(\beta_{r}\right),\left(\alpha, \beta_{r}\right)=1\right\}=\left\{\alpha \in \Delta^{+} \mid \operatorname{ht}(\alpha)<\operatorname{ht}\left(\beta_{r}^{\prime}\right),\left(\alpha, \beta_{r}^{\prime}\right)=1\right\} .
$$

Since \# $\left\{\alpha \in \Delta^{+} \mid \mathrm{ht}(\alpha)<\mathrm{ht}\left(\beta_{r}^{\prime}\right),\left(\alpha, \beta_{r}^{\prime}\right)=1\right\}=2(h-1)$ by our induction hypothesis, we obtain equation (B.3), as desired.

Case 2. Assume next that $\left(\beta_{r}^{\prime}, \alpha_{j_{l}}\right)=-1$, or equivalently, $\left(\beta_{r}, \alpha_{j_{l}}\right)=1$; in this case, $\beta_{r}=\beta_{r}^{\prime}+\alpha_{j_{l}}$, and hence ht $\left(\beta_{r}^{\prime}\right)=\operatorname{ht}\left(\beta_{r}\right)-1=h-1$. In addition, since $\beta_{l}=\alpha_{j_{l}}$, it follows that

$$
\begin{aligned}
\left\{r<t \leq l \mid\left(\beta_{t}, \beta_{r}\right)=1\right\} & =\left\{r<t \leq l-1 \mid\left(\beta_{t}, \beta_{r}\right)=1\right\} \cup\{l\} \\
& =\left\{r<t \leq l-1 \mid\left(s_{j_{l}} \beta_{t}, s_{j_{l}} \beta_{r}\right)=1\right\} \cup\{l\} \\
& =\left\{r<t \leq l-1 \mid\left(\beta_{t}^{\prime}, \beta_{r}^{\prime}\right)=1\right\} \cup\{l\} .
\end{aligned}
$$

By our induction hypothesis, we have \# $\left\{r<t \leq l-1 \mid\left(\beta_{t}^{\prime}, \beta_{r}^{\prime}\right)=1\right\}=h-2$, and hence $\#\{r<t \leq l \mid$ $\left.\left(\beta_{t}, \beta_{r}\right)=1\right\}=h-1$, as desired.

Let us prove equation (B.3) in this case. For simplicity of notation, we set

$$
\begin{aligned}
& R=R_{r}:=\left\{\alpha \in \Delta^{+} \mid \operatorname{ht}(\alpha)<\operatorname{ht}\left(\beta_{r}\right),\left(\alpha, \beta_{r}\right)=1\right\}, \\
& S=S_{r}:=\left\{\alpha \in \Delta^{+} \mid \operatorname{ht}(\alpha)<\operatorname{ht}\left(\beta_{r}^{\prime}\right),\left(\alpha, \beta_{r}^{\prime}\right)=1\right\} .
\end{aligned}
$$

Since $\left(\beta_{r}, \alpha_{j_{l}}\right)=1$ and $\beta_{r}-\alpha_{j_{l}}=s_{j_{l}} \beta_{r}=\beta_{r}^{\prime} \in \Delta^{+}$, we deduce that $\alpha_{j_{l}}, \beta_{r}-\alpha_{j_{l}} \in R$. We claim that

$$
s_{j_{l}} \alpha \in S \quad \text { for all } \alpha \in R \backslash\left\{\alpha_{j_{l}}, \beta_{r}-\alpha_{j_{l}}\right\} \text {. }
$$

It is easily verified that $s_{j_{l}} \alpha \in \Delta^{+}$and $\left(s_{j_{l}} \alpha, \beta_{r}^{\prime}\right)=1$. We show that ht $\left(s_{j_{l}} \alpha\right)<\mathrm{ht}\left(\beta_{r}^{\prime}\right)$. By Lemma B.1, $\left(\alpha, \alpha_{j_{l}}\right) \in\{0, \pm 1\}$. If $\left(\alpha, \alpha_{j_{l}}\right)=1$, then it is obvious that ht $\left(s_{j_{l}} \alpha\right)<\operatorname{ht}\left(\beta_{r}^{\prime}\right)$. Assume that $\left(\alpha, \alpha_{j_{l}}\right)=0$. Suppose, for a contradiction, that ht $\left(s_{j_{l}} \alpha\right) \geq \mathrm{ht}\left(\beta_{r}^{\prime}\right)$. Since ht $(\alpha)<\operatorname{ht}\left(\beta_{r}\right)$ and ht $\left(\beta_{r}^{\prime}\right)=\operatorname{ht}\left(\beta_{r}\right)-1$, we have ht $\left(s_{j_{l}} \alpha\right)=$ ht $\left(\beta_{r}^{\prime}\right)$. Since $\left(s_{j_{l}} \alpha, \beta_{r}^{\prime}\right)=1$, we see that $\beta_{r}^{\prime}-s_{j_{l}} \alpha \in \Delta$. However, ht $\left(\beta_{r}^{\prime}-s_{j_{l}} \alpha\right)=0$, which is a contradiction. Assume that $\left(\alpha, \alpha_{j_{l}}\right)=-1$; in this case, $s_{j_{l}} \alpha=\alpha+\alpha_{j_{l}}$. Suppose, for a contradiction, that ht $\left(s_{j_{l}} \alpha\right) \geq \mathrm{ht}\left(\beta_{r}^{\prime}\right)$; since ht $\left(s_{j_{l}} \alpha\right)=\mathrm{ht}(\alpha)+1$ and ht $\left(\beta_{r}^{\prime}\right)=\mathrm{ht}\left(\beta_{r}\right)-1$, and since $\mathrm{ht}(\alpha)<\mathrm{ht}\left(\beta_{r}\right)$, ht $\left(s_{j_{l}} \alpha\right)$ is equal to ht $\left(\beta_{r}^{\prime}\right)$ or ht $\left(\beta_{r}^{\prime}\right)+1$. By the same reasoning as before, we see that ht $\left(s_{j_{l}} \alpha\right) \neq$ ht $\left(\beta_{r}^{\prime}\right)$. Therefore, ht $\left(s_{j_{l}} \alpha\right)=\mathrm{ht}\left(\beta_{r}^{\prime}\right)+1$, and hence $\mathrm{ht}(\alpha)=\operatorname{ht}\left(\beta_{r}\right)-1$. Also, we see that $s_{\alpha} \beta_{r}=\beta_{r}-\alpha \in \Delta^{+}$. From these, we deduce that $\alpha=\beta_{r}-\alpha_{j}$ for some $j \in I$. It follows that

$$
-1=\left(\alpha, \alpha_{j_{l}}\right)=\left(\beta_{r}, \alpha_{j_{l}}\right)-\left(\alpha_{j}, \alpha_{j_{l}}\right)=1-\left(\alpha_{j}, \alpha_{j_{l}}\right),
$$


and hence $\left(\alpha_{j}, \alpha_{j_{l}}\right)=2$. This implies that $j=j_{l}$, and hence $\alpha=\beta_{r}-\alpha_{j_{l}}$, which is a contradiction. Thus, we have shown that ht $\left(s_{j_{l}} \alpha\right)<$ ht $\left(\beta_{r}^{\prime}\right)$ in all cases. Therefore, we obtain a map

$$
s_{j_{l}}: R \backslash\left\{\alpha_{j_{l}}, \beta_{r}-\alpha_{j_{l}}\right\} \rightarrow S, \quad \alpha \mapsto s_{j_{l}} \alpha .
$$

Similarly, we obtain its inverse

$$
s_{j_{l}}: S \rightarrow R \backslash\left\{\alpha_{j_{l}}, \beta_{r}-\alpha_{j_{l}}\right\}, \quad \alpha \mapsto s_{j_{l}} \alpha .
$$

Recall that ht $\left(\beta_{r}^{\prime}\right)=\mathrm{ht}\left(\beta_{r}\right)-1=h-1$. We conclude that

$$
\begin{aligned}
\# R & =\#\left(R \backslash\left\{\alpha_{j_{l}}, \beta_{r}-\alpha_{j_{l}}\right\}\right)+2 \\
& =\# S+2=2(h-2)+2=2(h-1),
\end{aligned}
$$

as desired. This proves the lemma.

Remark B.5. Keep the notation and setting as before. Set $1 \leq r \leq l$. We set

$$
R_{r}:=\left\{\alpha \in \Delta^{+} \mid \operatorname{ht}(\alpha)<\operatorname{ht}\left(\beta_{r}\right),\left(\alpha, \beta_{r}\right)=1\right\} .
$$

If $\alpha \in R_{r}$, then $s_{\alpha} \beta_{r}=\beta_{r}-\alpha \in \Delta^{+}$; it is easily verified that $\beta_{r}-\alpha \in R_{r}$. Hence $\sigma: \alpha \mapsto \beta_{r}-\alpha$ is an involution on $R_{r}$; notice that $\sigma$ is fixed-point-free (otherwise, $\beta_{r}=2 \alpha$ for some $\alpha \in R_{r}$ ). By Lemma B.3,

$$
B_{r}:=\left\{\beta_{t} \mid r<t \leq l,\left(\beta_{t}, \beta_{r}\right)=1\right\}
$$

is a subset of $R_{r}$; recall from Lemma B.4 that \# $B_{r}=h-1$ and $\# R_{r}=2(h-1)$, where $h:=\operatorname{ht}\left(\beta_{r}\right)$. We claim that $\sigma\left(B_{r}\right) \sqcup B_{r}=R_{r}$. Suppose, for a contradiction, that $\sigma\left(\beta_{t}\right)=\beta_{r}-\beta_{t} \in B_{r}$ for some $\beta_{t} \in B_{r}$. Let $r<s \leq l$ be such that $\sigma\left(\beta_{t}\right)=\beta_{s}$. Then $\beta_{r}=\beta_{s}+\beta_{t}$, and hence $\left(\lambda, \beta_{r}\right)=\left(\lambda, \beta_{s}\right)+\left(\lambda, \beta_{t}\right)=-2$ by Lemma B.2(1). However, this contradicts the assumption that $\lambda$ is minuscule. It follows that $\sigma\left(B_{r}\right) \cap$ $B_{r}=\emptyset$, and hence $\#\left(\sigma\left(B_{r}\right) \cup B_{r}\right)=2(h-1)=\# R_{r}$. Thus, we conclude that $\sigma\left(B_{r}\right) \sqcup B_{r}=R_{r}$.

We will prove equation (3.59). For $v \in\left\{w, s_{\beta_{r}} w\right\}$, we set

$$
\begin{aligned}
& X(v):=\left\{\gamma \in \operatorname{Inv}(v) \mid s_{\beta_{r}} \gamma \in \Delta^{+}\right\}, \\
& Y(v):=\left\{\gamma \in \operatorname{Inv}(v) \mid s_{\beta_{r}} \gamma \in \Delta^{-}\right\} .
\end{aligned}
$$

Note that $\operatorname{Inv}(v)=X(v) \sqcup Y(v)$ for $v \in\left\{w, s_{\beta_{r}} w\right\}$. We see that the map $X(w) \rightarrow X\left(s_{\beta_{r}} w\right), \gamma \mapsto s_{\beta_{r}} \gamma$, is bijective. Therefore,

$$
\ell(w)-\ell\left(s_{\beta_{r}} w\right)=\# Y(w)-\# Y\left(s_{\beta_{r}} w\right) .
$$

Also, by Lemma B.1 and the assumption that $s_{\beta_{r}} w<w$, it follows that

$$
Y(w)=\left\{\beta_{r}\right\} \sqcup\left(\operatorname{Inv}(w) \cap R_{r}\right), \quad Y\left(s_{\beta_{r}} w\right)=\operatorname{Inv}\left(s_{\beta_{r}} w\right) \cap R_{r}
$$

(for the definition of $R_{r}$, see Remark B.5). Recall from Remark B.5 that for $\beta_{t} \in B_{r}, \sigma\left(\beta_{t}\right)=\beta_{r}-\beta_{t} \in$ $R_{r} \backslash B_{r}$, and that $R_{r}=B_{r} \sqcup \sigma\left(B_{r}\right)$. Since $\beta_{r} \in \operatorname{Inv}(w)$, for each $\beta_{t} \in B_{r}$, one of the following holds:

(i) $\beta_{t}, \sigma\left(\beta_{t}\right) \in \operatorname{Inv}(w)$.

(ii) $\beta_{t} \in \operatorname{Inv}(w)$ and $\sigma\left(\beta_{t}\right) \notin \operatorname{Inv}(w)$.

(iii) $\beta_{t} \notin \operatorname{Inv}(w)$ and $\sigma\left(\beta_{t}\right) \in \operatorname{Inv}(w)$.

We see that these are equivalent, respectively, to the following:

(i)' $\beta_{t}, \sigma\left(\beta_{t}\right) \notin \operatorname{Inv}\left(s_{\beta_{r}} w\right)$.

(ii)' $\beta_{t} \in \operatorname{Inv}\left(s_{\beta_{r}} w\right)$ and $\sigma\left(\beta_{t}\right) \notin \operatorname{Inv}\left(s_{\beta_{r}} w\right)$.

(iii) $\beta_{t} \notin \operatorname{Inv}\left(s_{\beta_{r}} w\right)$ and $\sigma\left(\beta_{t}\right) \in \operatorname{Inv}\left(s_{\beta_{r}} w\right)$. 
Therefore, if we set

$$
\begin{aligned}
& a:=\#\left\{\beta_{t} \in B_{r} \mid \beta_{t}, \sigma\left(\beta_{t}\right) \in \operatorname{Inv}(w)\right\}, \\
& b:=\#\left\{\beta_{t} \in B_{r} \mid \beta_{t} \in \operatorname{Inv}(w), \sigma\left(\beta_{t}\right) \notin \operatorname{Inv}(w)\right\}, \\
& c:=\#\left\{\beta_{t} \in B_{r} \mid \beta_{t} \notin \operatorname{Inv}(w), \sigma\left(\beta_{t}\right) \in \operatorname{Inv}(w)\right\},
\end{aligned}
$$

we have

$$
\begin{aligned}
\# Y(w) & =1+\#\left(\operatorname{Inv}(w) \cap R_{r}\right)=1+2 a+b+c, \\
\# Y\left(s_{\beta_{r}} w\right) & =\#\left(\operatorname{Inv}\left(s_{\beta_{r}} w\right) \cap R_{r}\right)=b+c,
\end{aligned}
$$

and hence

$$
\ell(w)-\ell\left(s_{\beta_{r}} w\right)=\# Y(w)-\# Y\left(s_{\beta_{r}} w\right)=1+2 a .
$$

Now we see that

$$
\begin{aligned}
& \ell_{\lambda, r}^{-}(w)-\ell_{\lambda, r}^{-}\left(s_{\beta_{r}} w\right) \\
& \quad=\#\left(\operatorname{Inv}(w) \cap\left\{\beta_{r}, \beta_{r+1}, \ldots, \beta_{l}\right\}\right)-\#\left(\operatorname{Inv}\left(s_{\beta_{r}} w\right) \cap\left\{\beta_{r}, \beta_{r+1}, \ldots, \beta_{l}\right\}\right) \\
& \quad=\#\left(\left\{\beta_{r}\right\} \sqcup\left(\operatorname{Inv}(w) \cap\left\{\beta_{r+1}, \ldots, \beta_{l}\right\}\right)\right)-\#\left(\operatorname{Inv}\left(s_{\beta_{r}} w\right) \cap\left\{\beta_{r+1}, \ldots, \beta_{l}\right\}\right)=:(*),
\end{aligned}
$$

where the last equality follows from the assumption that $s_{\beta_{r}} w<w$. Also, we deduce that for $r<s \leq l$ with $\left(\beta_{s}, \beta_{r}\right)=0, \beta_{s} \in \operatorname{Inv}(w)$ if and only if $\beta_{s} \in \operatorname{Inv}\left(s_{\beta_{r}} w\right)$. Therefore,

$$
\begin{aligned}
(*) & =1+\#\left(\operatorname{Inv}(w) \cap B_{r}\right)-\#\left(\operatorname{Inv}\left(s_{\beta_{r}} w\right) \cap B_{r}\right) \\
& =1+(a+b)-b=1+a .
\end{aligned}
$$

Thus we conclude that

$$
2\left(\ell_{\lambda, r}^{-}(w)-\ell_{\lambda, r}^{-}\left(s_{\beta_{r}} w\right)\right)-1=2(1+a)-1=2 a+1 \stackrel{(B .7)}{=} \ell(w)-\ell\left(s_{\beta_{r}} w\right),
$$

as desired. This proves equation (3.59).

\section{B.2. Proofs of equations (3.58) and (3.60)}

Let $\omega: I \rightarrow I$ be the Dynkin diagram automorphism induced by the longest element $w_{\circ}-$ that is, $w_{\circ} \alpha_{i}=-\alpha_{\omega(i)}$ for $i \in I$; note that $\left\lfloor w_{\circ}\right\rfloor \varpi_{k}=w_{\circ} \varpi_{k}=-\varpi_{\omega(k)}$. We see that $\varpi_{\omega(k)}$ is also minuscule. In addition, $y^{-1}=s_{i_{m}} \cdots s_{i_{1}} \in W^{\omega(J)}=W^{I \backslash\{\omega(k)\}}$. We set $v:=y^{-1} \varpi_{\omega(k)}$; for $\gamma \in \Delta^{+}$,

$$
(\gamma, \lambda)=1 \Longleftrightarrow\left(\gamma, y^{-1}\left\lfloor w_{\circ}\right\rfloor \varpi_{k}\right)=1 \Longleftrightarrow\left(\gamma, y^{-1}\left(-\varpi_{\omega(k)}\right)\right)=1 \Longleftrightarrow(\gamma, v)=-1 .
$$

Hence it follows that $\operatorname{Inv}(w)_{\lambda}^{+}=\operatorname{Inv}(w)_{v}^{-}$for $w \in W$. Also, it is easily verified that $\ell_{\lambda, s}^{+}(w)=\ell_{\nu, s}^{-}(w)$ for all $w \in W$. Thus equations (3.58) and (3.60) follow from equations (3.57) and (3.59) (applied to $v$ ), respectively.

This completes the proof of Proposition 3.9.

Acknowledgements. The authors would like to thank Cristian Lenart, Leonardo Mihalcea and Mark Shimozono for helpful discussions. We also thank an anonymous referee for useful suggestions which improved our exposition. The first author was supported in part by Grant-in-Aid for JSPS Fellows 20J12058. The second author was supported in part by JSPS Grant-in-Aid for Scientific Research (B) 16H03920. The third author was supported in part by a Collaboration Grant for Mathematicians from the Simons Foundation (Award ID: 638577). The fourth author was supported in part by JSPS Grant-in-Aid for Scientific Research (C) $19 \mathrm{~K} 03415$.

Conflict of Interest: None. 


\section{References}

[1] A. Braverman and M. Finkelberg, 'Semi-infinite Schubert varieties and quantum $K$-theory of flag manifolds', J. Amer. Math. Soc. 27(4) (2014), 1147-1168.

[2] A. Braverman and M. Finkelberg, 'Weyl modules and $q$-Whittaker functions', Math. Ann. 359(1-2) (2014), $45-59$.

[3] F. Brenti, S. Fomin and A. Postnikov, 'Mixed Bruhat operators and Yang-Baxter equations for Weyl groups', Internat. Math. Res. Notices 1999 (8), 419-441.

[4] I. Cherednik and D. Orr, 'Nonsymmetric difference Whittaker functions', Math. Z. 279(3-4) (2015), 879-938.

[5] A. Givental and Y.-P. Lee, 'Quantum $K$-theory on flag manifolds, finite-difference Toda lattices and quantum groups', Invent. Math. 151(1) (2003), 193-219.

[6] R. M. Green, Combinatorics of Minuscule Representations, Cambridge Tracts in Mathematics vol. 199 (Cambridge University Press, Cambridge, 2013).

[7] S. Griffeth and A. Ram, 'Affine Hecke algebras and the Schubert calculus', European J. Combin. 25 (2004), $1263-1283$.

[8] B. Kostant and S. Kumar, 'T-equivariant $K$-theory of generalized flag varieties', J. Differential Geom. 32(2) (1990), 549-603.

[9] V. G. Kac, Infinite Dimensional Lie Algebras, 3rd edn (Cambridge University Press, Cambridge, 1990).

[10] S. Kato, 'Loop structure on equivariant $K$-theory of semi-infinite flag manifolds', Preprint, 2020, arXiv:1805.01718.

[11] S. Kato, 'Frobenius splitting of Schubert varieties of semi-infinite flag manifolds', Preprint, 2021, arXiv:1810.07106.

[12] S. Kato, 'On quantum $K$-groups of partial flag manifolds', Preprint, 2020, arXiv:1906.09343.

[13] S. Kato, S. Naito and D. Sagaki, 'Equivariant $K$-theory of semi-infinite flag manifolds and the Pieri-Chevalley formula', Duke Math. J. 169 (2020), 2421-2500.

[14] P. Koroteev, 'A-type quiver varieties and ADHM moduli spaces, Comm. Math. Phys. 381(1) (2021), $175-207$.

[15] P. Koroteev and A. M. Zeitlin, ‘qKZ/tRS duality via quantum $K$-theoretic counts', Preprint, 2019, arXiv:1802.04463.

[16] T. Kouno, S. Naito, D. Orr and D. Sagaki, 'Inverse $K$-Chevalley formulas for semi-infinite flag manifolds, II', In preparation.

[17] C. Lenart, 'A $K$-theory version of Monk's formula and some related multiplication formulas', J. Pure Appl. Algebra 179(1-2) (2003), 137-158.

[18] C. Lenart, 'On the combinatorics of crystal graphs. II. The crystal commutor', Proc. Amer. Math. Soc. 136(3) (2008), 825-837.

[19] C. Lenart, S. Naito and D. Sagaki, 'A general Chevalley formula for semi-infinite flag manifolds and quantum $K$-theory', Preprint, 2021, arXiv:2010.06143.

[20] C. Lenart and A. Postnikov, 'Affine Weyl groups in $K$-theory and representation theory', Int. Math. Res. Not. IMRN 2007(9) (2007), rnm038.

[21] C. Lenart and M. Shimozono, 'Equivariant $K$-Chevalley rules for Kac-Moody flag manifolds', Amer. J. Math. 136 (2014), 1175-1213.

[22] P. Littelmann and A. Seshadri, 'Pieri-Chevalley type formula for $K(G / B)$ and standard monomial theory', in Studies in Memory of Issai Schur, Progress in Mathematics vol. 210 (Birkhäuser, Boston, MA, 2003), 155-176.

[23] O. Mathieu, 'Positivity of some intersections in $K_{0}(G / B)$ ', Commutative algebra, homological algebra and representation theory (Catania/Genoa/Rome, 1998), J. Pure Appl. Algebra 152(1-3) (2000), 231-243.

[24] S. Naito, D. Orr and D. Sagaki, 'Chevalley formula for anti-dominant weights in the equivariant $K$-theory of semi-infinite flag manifolds', Adv. Math. 387 (2021), 107828.

[25] D. Orr, 'Equivariant $K$-theory of the semi-infinite flag manifold as a nil-DAHA module', Preprint, 2020, arXiv:2001.03490.

[26] H. Pittie and A. Ram, 'A Pieri-Chevalley formula in the $K$-theory of a $G / B$-bundle', Electron. Res. Announc. Amer. Math. Soc. 5 (1999), 102-107.

[27] J. R. Stembridge, 'Combinatorial models for Weyl characters', Adv. Math. 168(1) (2002), 96-131. 